\title{
Zinc-Induced Effects on Productivity, Zinc Use Efficiency, and Grain Biofortification of Bread Wheat under Different Tillage Permutations
}

\author{
Usman Zulfiqar ${ }^{1}\left(\mathbb{D}\right.$, Saddam Hussain ${ }^{1, *(\mathbb{D}, \text { Muhammad Ishfaq }}{ }^{1}$, Amar Matloob $^{2}$, Nauman Ali ${ }^{3}$, \\ Muhammad Ahmad ${ }^{1}$, Mohammed Nasser Alyemeni ${ }^{4}$ and Parvaiz Ahmad ${ }^{4,5, *(D)}$ \\ 1 Department of Agronomy, Faculty of Agriculture, University of Agriculture, Faisalabad 38040, Pakistan; \\ usmanzulfiqar2664@gmail.com (U.Z.); ishfaq2727@gmail.com (M.I.); ahmadbajwa516@gmail.com (M.A.) \\ 2 Department of Agronomy, Faculty of Agriculture \& Environmental Sciences, Muhammad Nawaz Shareef \\ University of Agriculture, Multan 60000, Pakistan; amar.matloob@mnsuam.edu.pk \\ 3 Agronomic Research Institute, Ayub Agricultural Research Institute, Faisalabad 38000, Pakistan; \\ naumanalich22@gmail.com \\ 4 Botany and Microbiology Department, College of Science, King Saud University, Riyadh 11451, \\ Saudi Arabia; mnyemeni@ksu.edu.sa \\ 5 Department of Botany, S.P. College, Srinagar, Jammu and Kashmir, 190001, India \\ * Correspondence: sadamhussainuaf@gmail.com or shussain@uaf.edu.pk (S.H.); \\ pahmad@ksu.edu.sa or parvaizbot@yahoo.com (P.A.)
}

Received: 7 September 2020; Accepted: 2 October 2020; Published: 14 October 2020 updates

\begin{abstract}
Zinc (Zn) deficiency is a global concern for human health and causes a decrease in crop production and nutritional characteristics. A two-year field study was planned to evaluate comparative effects of various $\mathrm{Zn}$ application approaches in bread wheat under plough tillage (PT) and zero tillage (ZT) system. Cultivation of wheat under ZT improved the soil organic carbon (17\%), total soil porosity $(11 \%)$, soil microbial biomass nitrogen $(5 \%)$, and carbon (5\%) in comparison to PT system averaged across the two years. Various efficiency indices were significantly influenced by $\mathrm{Zn}$ application methods during both years of experimentation. However, grain $\mathrm{Zn}$ contents were maximum with foliar-applied Zn in PT (31\%) and soil-applied Zn under the ZT system (29.85\%). Moreover, $\mathrm{Zn}$ use also enhanced the bioavailable $\mathrm{Zn}$ as lower phytate contents and phytate to $\mathrm{Zn}$ molar ratio were recorded. The highest bioavailable $\mathrm{Zn}$ was calculated for foliar (30\%) and soil application (28\%). Under both tillage systems, the maximum net benefits were obtained through Zn seed priming; nevertheless, ZT resulted in higher net benefits than PT due to low associated costs. In conclusion, $\mathrm{Zn}$ nutrition through different methods enhanced the productivity, profitability, and grain biofortification of wheat under PT and ZT systems.
\end{abstract}

Keywords: agronomic biofortification; micronutrient application methods; seed enhancements; Zn deficiency; Zinc enriched wheat

\section{Introduction}

Staple cereals (wheat, rice, and maize) are the principal source of food in developing countries with low amounts of micronutrients including zinc ( $\mathrm{Zn})$, boron (B), and iron (Fe) [1]. Therefore, the use of only staple food in daily diet is a major cause of widespread micronutrient deficiency in under-develop countries [2]. Wheat occupies a central position for the provision of micronutrients and $70 \%$ daily calories in third world countries [3,4]. Among micronutrients, deficiency of $\mathrm{Zn}$ is most common and widespread in wheat-growing regions. Worldwide, about $50 \%$ of cultivated soils are found deficient in bioavailable $\mathrm{Zn}$ [5]. The problem of $\mathrm{Zn}$ deficiency is associated with poor availability 
and higher $\mathrm{Zn}$ adsorption on soil particles owing to the higher $\mathrm{pH}$ and calcareous nature of soils [6]. Additionally, a considerable decline in yield due to insufficient $\mathrm{Zn}$ supply from the soil also lowers the $\mathrm{Zn}$ concentration in grains [7]. For example, $\mathrm{Zn}$ contents of wheat grains were reduced by $50 \%$ when it was cultivated on a Zn-deficient soil in Turkey [5].

More than two billion people worldwide are suffering from several disorders due to deficiency of micronutrients [8]. However, about 1.25 billion population is at the risk of $\mathrm{Zn}$ inadequacy [8,9]. In Pakistan, 40\% mothers and 33\% children are under $\mathrm{Zn}$ malnutrition particularly in rural communities [10]. About $49 \%$ of soils in the world [11] and 70\% of soils across Pakistan are $\mathrm{Zn}$ deficient [12]. $\mathrm{Zn}$ is an important micronutrient for biological systems in humans, animals, and plants. In plants, it has a central role in the integrity of biological membranes, enzyme activation, and protein synthesis [13,14].

Intensive and continuous wheat and rice cultivation has endangered the productivity and sustainability of rice-wheat cropping system (RWCS) owing to deteriorated soil heath, persistent minerals mining [15], micronutrient inadequacy [1,16], and higher production costs [17-19]. Timely sowing of wheat in conventional RWCS is delayed under plough tillage (PT). Moreover, continuous soil disturbance along with the cultivation of high yielding cultivars (that demand high levels of inputs) has caused a significant decline in system productivity owing to poor soil health and Zn deficiency [20]. Additionally, tillage affects the transformation and availability of $\mathrm{Zn}$ predominately in the top soil profile layers and plants [21]. Zero tillage (ZT) in this regard offers a practical solution for sustaining the productivity of RWCS [22]. It ensures wheat sowing at proper time, saves energy resources, and mends soil health as well as fertility $[19,23]$. Moreover, under ZT system, surface stratification of $\mathrm{Zn}$ is different from conventional tillage system. The higher amount of $\mathrm{Zn}$ leads to mount up in rhizosphere soil due to residues decay under ZT system as compared to the PT system [24]. Residues retention on soil surface improves the moisture retention capability, regulates the soil $\mathrm{pH}$ and temperature, and enhances the $\mathrm{Zn}$ availability to plants [25].

The $\mathrm{Zn}$ malnutrition can be minimized by various remedies like fortification of food, supplementation, diversification in diet, and biofortification [26]. From these methods, biofortification is a feasible and relatively inexpensive option, as it can be easily disseminated in remote areas of under-developed countries. It is a process of intentionally enhancing the nutritional value of edible parts of plants via genetic engineering or agronomic interventions [27]. Genetic biofortification is a less economical and time-consuming approach [28]. Biofortification via agronomic interventions (application of $\mathrm{Zn}$ fertilizer) is an easy and practical approach to produce $\mathrm{Zn}$ enriched grains [29]. It can be applied as seed treatments and basal as well as foliar application [30].

Role of $\mathrm{Zn}$ in enhancing the crop productivity and grain $\mathrm{Zn}$ concentration of bread wheat is well reported [31-33]. However, information on the comparative efficacy of $\mathrm{Zn}$ application methods in enhancing the productivity, profitability, grain biofortification, and bio-availability of wheat sown in traditional, as well as conservational tillage systems, is lacking. Therefore, the recent study was carried out with the hypothesis that $\mathrm{Zn}$ application would enhance the productivity and grain $\mathrm{Zn}$ concentration of bread wheat under different tillage systems. The objectives of this study were: (a) to check the most appropriate and affordable approach of $\mathrm{Zn}$ application to increase wheat productivity and grain $\mathrm{Zn}$ contents under PT and ZT, and (b) to examine the variations between PT and ZT regarding Zn use efficiency and grain biofortification.

\section{Materials and Methods}

\subsection{Experimental Site, Climate, and Soil}

This experiment was executed at Student Research Farm, University of Agriculture, Faisalabad (latitude $73.89^{\circ} \mathrm{E}$, longitude $31.62^{\circ} \mathrm{N}$, and altitude $183.8 \mathrm{~m}$ asl) for two consecutive growing seasons (2017-2018 and 2018-2019). The climate of study site (Faisalabad) is sub-tropical and has a dry climate with temperatures varying from $26^{\circ} \mathrm{C}$ to $38^{\circ} \mathrm{C}$ in the summer and $7^{\circ} \mathrm{C}$ to $20^{\circ} \mathrm{C}$ in the winter and the average rainfall 
is $350 \mathrm{~mm}$ (annually). For the entire period of experiment, the weather data were obtained from the Meteorological Cell, University of Agriculture, Faisalabad (Table 1), which is located very close to the experimental site. The experimental soil belonged to the Lyallpur soil series and classified as Haplic Yermosol in the classification of Food and Agriculture Organization (FAO) [34] and aridisol-fine silty hyperthermic Ustalfic, Haplagrid under United State Department of Agriculture (USDA) system of soil classification [35]. Pre-sowing soil analysis depicted that texture of experimental soil was sandy clay loam with $\mathrm{pH} 7.4$, electrical conductivity (EC) $0.51 \mathrm{dS} \mathrm{m}^{-1}$, total soil organic matter $0.66 \%$, total nitrogen (N) $0.059 \%$, available phosphorus (P) $7.3 \mathrm{mg} \mathrm{kg}^{-1}$, extractable potassium (K) $92 \mathrm{mg} \mathrm{kg}^{-1}$, and diethylene triamine pentaacetic acid (DTPA) extractable $\mathrm{Zn} 0.67 \mathrm{mg} \mathrm{kg}^{-1}$.

Table 1. Weather data of experimental site for the wheat season of 2017-2018 and 2018-2019.

\begin{tabular}{|c|c|c|c|c|c|c|c|c|c|c|c|c|}
\hline \multirow{3}{*}{ Months } & \multirow{2}{*}{\multicolumn{2}{|c|}{$\begin{array}{c}\text { Rainfall } \\
(\mathrm{mm})\end{array}$}} & \multirow{2}{*}{\multicolumn{2}{|c|}{$\begin{array}{c}\text { Relative Humidity } \\
(\%)\end{array}$}} & \multicolumn{6}{|c|}{ Temperature $\left({ }^{\circ} \mathrm{C}\right)$} & \multicolumn{2}{|c|}{ Sunshine (h) } \\
\hline & & & & & \multicolumn{2}{|c|}{ Monthly Maximum } & \multicolumn{2}{|c|}{ Monthly Minimum } & \multicolumn{2}{|c|}{ Monthly Mean } & \multirow[b]{2}{*}{ 2017-2018 } & \multirow[b]{2}{*}{ 2018-2019 } \\
\hline & $2017-2018$ & 2018-2019 & 2017-2018 & 2018-2019 & 2017-2018 & 2018-2019 & 2017-2018 & 2018-2019 & 2017-2018 & 2018-2019 & & \\
\hline November & 01.50 & 00.6 & 84.6 & 74.6 & 24.1 & 27.0 & 11.8 & 12.4 & 18.0 & 19.7 & 3.7 & 6.9 \\
\hline December & 04.20 & 00.7 & 69.3 & 81.7 & 22.0 & 21.7 & 6.7 & 6.5 & 14.4 & 14.1 & 6.0 & 6.9 \\
\hline January & 00.00 & 18.0 & 75.9 & 81.0 & 21.5 & 19.2 & 5.5 & 7.0 & 13.5 & 13.2 & 6.4 & 5.4 \\
\hline February & 09.50 & 73.2 & 73.3 & 79.0 & 24.0 & 20.3 & 9.5 & 9.1 & 16.7 & 14.7 & 6.5 & 6.7 \\
\hline March & 12.50 & 55.7 & 61.6 & 68.8 & 31.2 & 26.0 & 16.4 & 13.8 & 23.8 & 19.9 & 8.6 & 8.9 \\
\hline April & 07.90 & 31.2 & 47.3 & 57.4 & 36.8 & 35.0 & 20.8 & 20.6 & 28.8 & 27.8 & 9.1 & 9.0 \\
\hline
\end{tabular}

Source: Meteorological Cell, University of Agriculture Faisalabad, Pakistan. All the values of sunshine, relative humidity, and mean temperature are the monthly averages.

\subsection{Plant Material}

Seed of wheat cv. Anaaj-2017 was acquired from Wheat Research Institute, Faisalabad. Moisture contents and germination percentage of procured seeds was determined following ISTA, 2015 [36], and was $10.2 \%$ and $90 \%$, respectively.

\subsection{Experimentation}

The study consisted of two variables, namely, tillage systems (plough tillage; PT and zero tillage; ZT) and Zn application methods (No application, Zn seed coating, hydro-priming, Zn seed priming, $\mathrm{Zn}$ soil application, water foliar spray, and Zn foliar spray). In the PT system, the seedbed was conventionally prepared with three cultivations followed by plankings. After that, seed was sown with tractor-mounted seed-cum fertilizer drill. Wheat seeds were directly seeded into stubbles of preceding rice crop with ZT drill and no preparatory tillage operations were practiced in ZT system. During both study years, sowing of wheat was done in rows $(22.5 \mathrm{~cm}$ spaced), while the seeding rate was $125 \mathrm{~kg} \mathrm{ha}^{-1}$ under both tillage systems. Wheat seed was sown on 19 November in 2017 and on 28 November in 2018. For all treatments, $\mathrm{ZnSO}_{4} .7 \mathrm{H}_{2} \mathrm{O}$ was used as source of $\mathrm{Zn}$ and applied using different methods. For seed coating, a sticking solution was prepared using Arabic gum as a sticking agent, $1.25 \mathrm{~g} \mathrm{Zn} \mathrm{kg}^{-1}$ seed was added in sticky solution, and wheat seeds were dipped in this solution for $45 \mathrm{~min}$ and allowed to adhere the $\mathrm{Zn}$ solution on wheat seeds. Wheat seeds were soaked in distilled water (hydro-priming) or $0.25 \mathrm{M}$ aerated $\mathrm{Zn}$ solution (Zn priming) for $12 \mathrm{~h}$ with 1:5 seed weight to solution volume ratio for seed priming. Artificial aeration was given to seeds with an aquarium pump during soaking. After removal of seeds from the priming solution, their washing was done with distilled water and dried by forced air under shade until their initial weight. Application of $\mathrm{Zn}$ was done as a basal dose at $10 \mathrm{~kg} \mathrm{ha}^{-1}$ during seedbed preparation as soil application. For foliar application, $0.5 \% \mathrm{Zn}$ solution or water spray (distilled water) was applied with manual sprayer at the booting stage (BBCH code 40) [37]. Hydro-priming and water spray were considered as a positive control for $\mathrm{Zn}$ priming and $\mathrm{Zn}$ foliar spray, respectively. Rice sowing was done as a subsequent crop after wheat. For the last three years, the experimental patch had been under a rice-wheat rotation. For the study, a randomized complete block design under split plot arrangement was used. In the main plots, tillage methods were assigned, while $\mathrm{Zn}$ application approaches were placed in sub-plots. All the treatments were replicated thrice. Based on soil analysis, soil fertilization was carried out at N:P:K 100:85:65 kg 
ha ${ }^{-1}$ applying urea $(46 \% \mathrm{~N})$, di-ammonium phosphate (DAP) $\left(46 \% \mathrm{P}_{2} \mathrm{O}_{5}\right)$, and sulfate of potash (SOP) $\left(50 \% \mathrm{~K}_{2} \mathrm{O}\right)$. The whole quantity of $\mathrm{P}$ and $\mathrm{K}$ and $\frac{1}{2}$ of $\mathrm{N}$ was used during the time of sowing, whereas the remaining $\frac{1}{2}$ of $\mathrm{N}$ was top-dressed in two halves at first and second irrigation. Pre-sowing irrigation (soaking irrigation) was applied before sowing of wheat during both years of experimentation, whereas during the crop growth period, five irrigations (each $75 \mathrm{~mm}$ ) were applied using canal water as a source of irrigation. Wheat seeds were treated using Hombre ${ }^{\circledR} 37.25 \%$ FS (Imidacloprid $360 \mathrm{~g} \mathrm{~L}^{-1}$ and Tebuconazole $12.5 \mathrm{~g} \mathrm{~L}^{-1}$ ) at a rate of $2 \mathrm{~mL} \mathrm{~kg}^{-1}$ seed to control seed-borne and soil-borne pathogens. For weed control, Total ${ }^{\circledR} 80 \mathrm{WG}$ (Sulfosulfuron + Metasulfuron $16 \mathrm{~g}$ a.i. ha ${ }^{-1}$ ) was sprayed at 30 days after sowing. Wheat crop during the first and second season of experimentation was harvested on 14 April 2018, and 20 April 2019, respectively. The harvested wheat crop from each experimental unit was tangled into bundles and sundried for seven days; then, it was threshed using a mini-thresher.

\subsection{Data Recording}

\subsubsection{Soil Properties}

After wheat harvesting, random soil sampling (at 0-10 cm and 10-20 cm sampling depth) was done at three test sites of each experimental unit during both years of experimentation. The physical properties of soil were determined in the field including soil bulk density (SBD) using the core method of Blake and Hartge [38], the total soil porosity (TSP) by following Vomocil [39] procedures, and soil penetration resistance using the cone penetrometer. Total $\mathrm{N}$, available $\mathrm{P}$, and extractable $\mathrm{K}$ were determined according to the methods suggested by Bremner and Mulvaney [40], Olsen [41], and Richards [42], respectively. In addition, soil organic carbon (SOC) is estimated in accordance with Walkley and Black [43]. For determination of soil microbial biomass carbon (SMBC) and nitrogen (SMBN), samples of soil were collected at anthesis stage (BBCH code 69) [37] and chloroform fumigation extraction procedure was followed to determine the SMBC and SMBN, as per Brookes et al. [44] and Anderson and Ingram [45].

\subsubsection{Yield Attributes}

For calculating the number of productive tillers, data were recorded from three random spots $(1 \mathrm{~m} \times 1 \mathrm{~m})$ from each experimental plot at the harvest maturity. To determine the grains per spike, 20 spikes from each experimental unit were clipped and threshed manually. Manually harvested crop was tangled up in bundles and kept in the air for sun drying. Biological yield $\left(\mathrm{Mg} \mathrm{ha}^{-1}\right)$ was determined by weighing the sundried and tied bundles of harvested crop after seven days of harvesting. Each plot was threshed separately with the help of mini thresher. Grains were separated from chaff and straw and grain yield $\left(\mathrm{Mg} \mathrm{ha}^{-1}\right)$ was noted for each plot. For the 1000-grain weight determination, three sub-samples of 1000 grains for each experimental unit were drawn, counted, and weighed with a digital weighing balance. Harvest index was computed as a ratio of grain yield to biological yield and given in percentage.

\subsubsection{Grain and Straw Zn Concentration}

Samples of grain and straw were taken at the final harvest and prepared by the process of wet ashing [46]. Samples were kept in an oven (UF1060plus Memmert, Germany) at $70{ }^{\circ} \mathrm{C}$, and oven-dried samples were ground and weighed. Then, these samples were added in a di-acid $\left(\mathrm{HClO}_{4}: \mathrm{HNO}_{3}\right.$ at 3:10 $v / v$ ratio) mixture for the digestion process and placed on a digestion plate (Heidolph, USA model, MR3003). Afterward, the atomic absorption spectrophotometer (Shimadzu, UV-1201, Kyoto, Japan) was used to determine $\mathrm{Zn}$ concentrations in grain and straw samples.

\subsubsection{Estimation of Phytate Contents and Bioavailable $\mathrm{Zn}$}

For the determination of phytate in wheat grain, the procedure of Haug and Lantzsch [47] was followed with minor modifications, as described in Rehman et al. [48,49]. For phytate determination, 
ground seed samples $(0.1 \mathrm{~g})$ were placed in a tube along with $\mathrm{Na}_{2} \mathrm{SO}_{4}(10 \mathrm{~mL})(10 \%$ solution dissolved in $0.4 \mathrm{M} \mathrm{HCl}$ ) and stirred for three $\mathrm{h}$ and centrifuged (Sigma GmbH, Germany model. 2-16P) $4600 \mathrm{~g}$ for $20 \mathrm{~min}$. Afterward, $1 \mathrm{~mL}$ from the centrifuged sample was collected and mixed in ferric solution and heating was done at $95^{\circ} \mathrm{C}$ for $30 \mathrm{~min}$. Then, heated samples were instantly kept in cold water to reduce the temperature. Samples were again centrifuged for $20 \mathrm{~min}$ and $1 \mathrm{~mL}$ supernatant was added to fresh tube and mixed with $3 \mathrm{~mL}$ of 2,2'-bi-pyridine solution $\left(\mathrm{C}_{10} \mathrm{H}_{8} \mathrm{~N}_{2}\right)(5 \mathrm{~g}$ dissolved in $500 \mathrm{~mL}$ water with $1 \% v / v$ thioglycollic acid $\left(\mathrm{HSCH}_{2} \mathrm{COOH}\right)$ ). After the development of pink color, measurement was taken at $519 \mathrm{~nm}$ with an atomic absorption spectrophotometer against phytic acid standards. For calculation of [phytae]: [Zn], molar concentration of $\mathrm{Zn}$ and phytate in wheat grain was used. Bioavailable $\mathrm{Zn}$ was estimated by employing the trivariate model of $\mathrm{Zn}$ absorption [50]:

$$
\mathrm{TAZ}=0.5 \cdot\left[\mathrm{A}_{\mathrm{MAX}}+\mathrm{TDZ}+\mathrm{K}_{\mathrm{R}} \cdot\left(1+\frac{\mathrm{TDP}}{\mathrm{K}_{\mathrm{P}}}\right)-\sqrt{\left(\mathrm{A}_{\mathrm{MAX}}+\mathrm{TDZ}+\mathrm{K}_{\mathrm{R}} \cdot\left(1+\frac{\mathrm{TDP}}{\mathrm{K}_{\mathrm{P}}}\right)\right)^{2}-4 \cdot \mathrm{A}_{\mathrm{MAX}}+\mathrm{TDZ}}\right]
$$

where TAZ is the total absorbed $\mathrm{Zn}$ per day $\left(\mathrm{mg} \mathrm{day}^{-1}\right), \mathrm{TDZ}$ is total $\mathrm{Zn}$ in diet per day $\left(\mathrm{mmol} \mathrm{day}^{-1}\right)$, and TDP is total phytate contents in diet per day $\left(\mathrm{mmol} \mathrm{day}^{-1}\right)$. Moreover, there are three constants in the equation, i.e., maximum $\mathrm{Zn}$ absorption $\left(\mathrm{A}_{\mathrm{MAX}}=0.091\right)$, dissociation constants of $\mathrm{Zn}$-receptor binding reaction $\left(K_{R}=0.680\right)$, and phytate- $Z n$ binding reaction $\left(K_{P}=0.033\right)$ [51]. According to the FAO [52], the average daily consumption of wheat is $300 \mathrm{~g}$. Therefore, TAZ was measured for wheat flour (300 g) and presented as estimated bioavailable $\mathrm{Zn}$.

\subsubsection{Estimation of Zn Use Efficiency}

The estimated values of $\mathrm{Zn}$ use efficiencies were computed by following the formulas suggested by Fageria [53] and Shivay and Prasad [54]:

$$
\begin{aligned}
& \text { Agronomic efficiency }(\mathrm{AgE})=\frac{\mathrm{GY}_{\mathrm{Zn}}-\mathrm{GY}_{\mathrm{C}}}{\mathrm{Zn}_{\mathrm{a}}} \\
& \text { Physiological efficiency }(P E)=\frac{Y_{Z n}-Y_{C}}{U_{Z n}-U_{C}} \\
& \text { Agro - physiological efficiency }(\mathrm{AgPE})=\frac{\mathrm{GY}_{\mathrm{Zn}}-\mathrm{GY}_{\mathrm{C}}}{\mathrm{U}_{\mathrm{Zn}}-\mathrm{U}_{\mathrm{C}}} \\
& \text { Apparent recovery efficiency }(A R E)=\frac{U_{Z n}-U_{C}}{Z_{a}} \\
& \text { Utilization efficiency (UE) }=\mathrm{PE} \times \mathrm{ARE} \\
& \text { Partial factor productivity }(\mathrm{PFP})=\frac{\mathrm{GY}_{\mathrm{Zn}}}{\mathrm{Zn}_{\mathrm{a}}}
\end{aligned}
$$

where $G Y_{Z n}$ is the grain yield of $Z n$ treated plots, $G Y_{C}$ is the yield of untreated plots, $Z n_{a}$ is the total amount of $Z n$ applied, $Y_{Z n}$ is the grain and straw yield of $Z n$ treated plots, $Y_{C}$ is the grain and straw yield of untreated plots, $U_{Z n}$ is the $Z n$ uptake in grain and straw of $Z n$ treated plots, and $U_{C}$ is the $Z n$ uptake in grain and straw of untreated plots.

\subsubsection{Economic Analysis}

For estimation of the economic viability of tillage systems as well as $\mathrm{Zn}$ application methods, economic analyses were performed [55]. Actual grain and straw yield of wheat crop were reduced to $10 \%$ for adjusting grain and straw yield as per farmer's level, as there are more precise management practices in experimental research as compared to farmer fields. Seed, fertilizer, irrigation, plant protection measures, harvesting, threshing, and labor costs were considered as fixed cost and remained the same for all treatments. The cost incurred for tillage practices and $\mathrm{Zn}$ application was included as a variable 
cost. Net benefit was calculated by deducting gross income from the total cost. The benefit-cost ratio (BCR) was computed as a ratio of gross income and total cost.

\subsubsection{Statistical Analysis}

Data on all parameters were analyzed statistically by performing analysis of variance (ANOVA) technique through statistical software Statistix 10.1 (Analytical Software, Statistix; Tallahassee, FL, USA, 1985-2003). Tukey's HSD (honestly significant difference) at 5\% probability level was used for comparison of treatment's means [56].

\section{Results}

\subsection{Soil Properties}

Tillage systems considerably affected the soil physical and biological properties (SBD, TSP, PR, SMBC, SMBN, and SOC) and nutrient status (total N, available $\mathrm{P}$, and extractable $\mathrm{K}$ ), whereas soil properties remained unaffected with Zn nutrition. Averaged across two years, SBD was $4.40 \%$ higher at $0-10 \mathrm{~cm}$, and $3.80 \%$ higher at 10-20 cm depth under PT as compared to ZT (Table 2). However, ZT system recorded higher values for TSP (14.97\% and 7.28\%), PR $(9.30 \%$ and $15.30 \%)$, SMBC $(5.15 \%$ and $4.39 \%)$, SMBN (4.6\% and $5.11 \%)$, and SOC (16.56\% and $16.55 \%)$ at $0-10 \mathrm{~cm}$ and $10-20 \mathrm{~cm}$ depth, respectively, as compared to ZT (Table 2). Total $\mathrm{N}$ was $7.70 \%$ and $8.33 \%$ higher under ZT during the first and second experimental year, respectively (Figure 1a; Table S1). Similarly, 3.73\% and 6.02\% higher available P was observed under ZT than PT during 2017-2018 and 2018-2019, respectively (Figure 1b; Table S1). Extractable K was statistically unaffected by the tillage systems during the first year; however, the ZT system showed higher value (3.06\%) for extractable K as compared to PT system during 2018-2019 (Figure 1c; Table S1).

Table 2. Influence of tillage systems on soil health parameters recorded after wheat harvest.

\begin{tabular}{|c|c|c|c|c|}
\hline \multirow{2}{*}{ Treatments } & \multicolumn{2}{|c|}{ 2017-2018 } & \multicolumn{2}{|c|}{ 2018-2019 } \\
\hline & $0-10 \mathrm{~cm}$ & $10-20 \mathrm{~cm}$ & $0-10 \mathrm{~cm}$ & $10-20 \mathrm{~cm}$ \\
\hline \multicolumn{5}{|c|}{ Soil bulk density $\left(\mathrm{g} \mathrm{cm}^{-3}\right)$} \\
\hline Plough tillage & $1.67 \mathrm{~A}$ & $1.63 \mathrm{~A}$ & $1.65 \mathrm{~A}$ & $1.66 \mathrm{~A}$ \\
\hline Zero tillage & $1.60 \mathrm{~B}$ & $1.57 \mathrm{~B}$ & $1.58 \mathrm{~B}$ & $1.59 \mathrm{~B}$ \\
\hline $\operatorname{HSD}(p \leq 0.05)$ & 0.04 & 0.03 & 0.03 & 0.02 \\
\hline \multicolumn{5}{|c|}{ Total Soil Porosity (\%) } \\
\hline Plough tillage & $37.75 \mathrm{~B}$ & $39.00 \mathrm{~B}$ & $36.67 \mathrm{~B}$ & $38.00 \mathrm{~B}$ \\
\hline Zero tillage & $43.57 \mathrm{~A}$ & $41.85 \mathrm{~A}$ & $42.00 \mathrm{~A}$ & $40.76 \mathrm{~A}$ \\
\hline $\operatorname{HSD}(p \leq 0.05)$ & 0.90 & 0.63 & 0.70 & 0.38 \\
\hline \multicolumn{5}{|c|}{ Penetration Resistance (kPa) } \\
\hline Plough tillage & $513.1 \mathrm{~A}$ & $580.1 \mathrm{~B}$ & $520.4 \mathrm{~B}$ & $575.4 \mathrm{~B}$ \\
\hline Zero tillage & $530.9 \mathrm{~A}$ & $640.9 \mathrm{~A}$ & $599.1 \mathrm{~A}$ & $691.1 \mathrm{~A}$ \\
\hline $\operatorname{HSD}(p \leq 0.05)$ & NS & 13.61 & 19.51 & 11.63 \\
\hline \multicolumn{5}{|c|}{ Soil Microbial Biomass Carbon $\left(\mu \mathrm{g} \mathrm{g}^{-1}\right)$} \\
\hline Plough tillage & $160.8 \mathrm{~B}$ & $163.4 \mathrm{~B}$ & $168.4 \mathrm{~B}$ & $164.9 \mathrm{~B}$ \\
\hline Zero tillage & $168.9 \mathrm{~A}$ & $169.5 \mathrm{~A}$ & $177.3 \mathrm{~A}$ & $173.3 \mathrm{~A}$ \\
\hline $\operatorname{HSD}(p \leq 0.05)$ & 1.41 & 2.92 & 3.41 & 1.68 \\
\hline
\end{tabular}


Table 2. Cont.

\begin{tabular}{ccccc}
\hline \multicolumn{5}{c}{ Soil Microbial Biomass Nitrogen $\left(\boldsymbol{\mu g ~ \mathbf { ~ } ^ { \mathbf { - 1 } } )}\right.$} \\
\hline Plough tillage & $574.9 \mathrm{~B}$ & $562.0 \mathrm{~B}$ & $578.0 \mathrm{~B}$ & $561.1 \mathrm{~B}$ \\
Zero tillage & $607.6 \mathrm{~A}$ & $587.3 \mathrm{~A}$ & $598.4 \mathrm{~A}$ & $593.3 \mathrm{~A}$ \\
HSD $(p \leq 0.05)$ & 11.96 & 2.21 & 8.69 & 5.32 \\
\hline \multicolumn{5}{c}{ Soil Organic Carbon $\left.\mathbf{( g ~ k g}^{\mathbf{- 1}}\right)$} \\
\hline Plough tillage & $6.09 \mathrm{~B}$ & $5.62 \mathrm{~B}$ & $6.58 \mathrm{~B}$ & $5.73 \mathrm{~B}$ \\
Zero tillage & $7.21 \mathrm{~A}$ & $6.55 \mathrm{~A}$ & $7.55 \mathrm{~A}$ & $6.68 \mathrm{~A}$ \\
HSD $(p \leq 0.05)$ & 0.11 & 0.50 & 0.18 & 0.05 \\
\hline
\end{tabular}

Means sharing the same uppercase letter during a year for a parameter do not differ significantly at $p \leq 0.05$ honestly significant difference (HSD); NS = Non-significant.

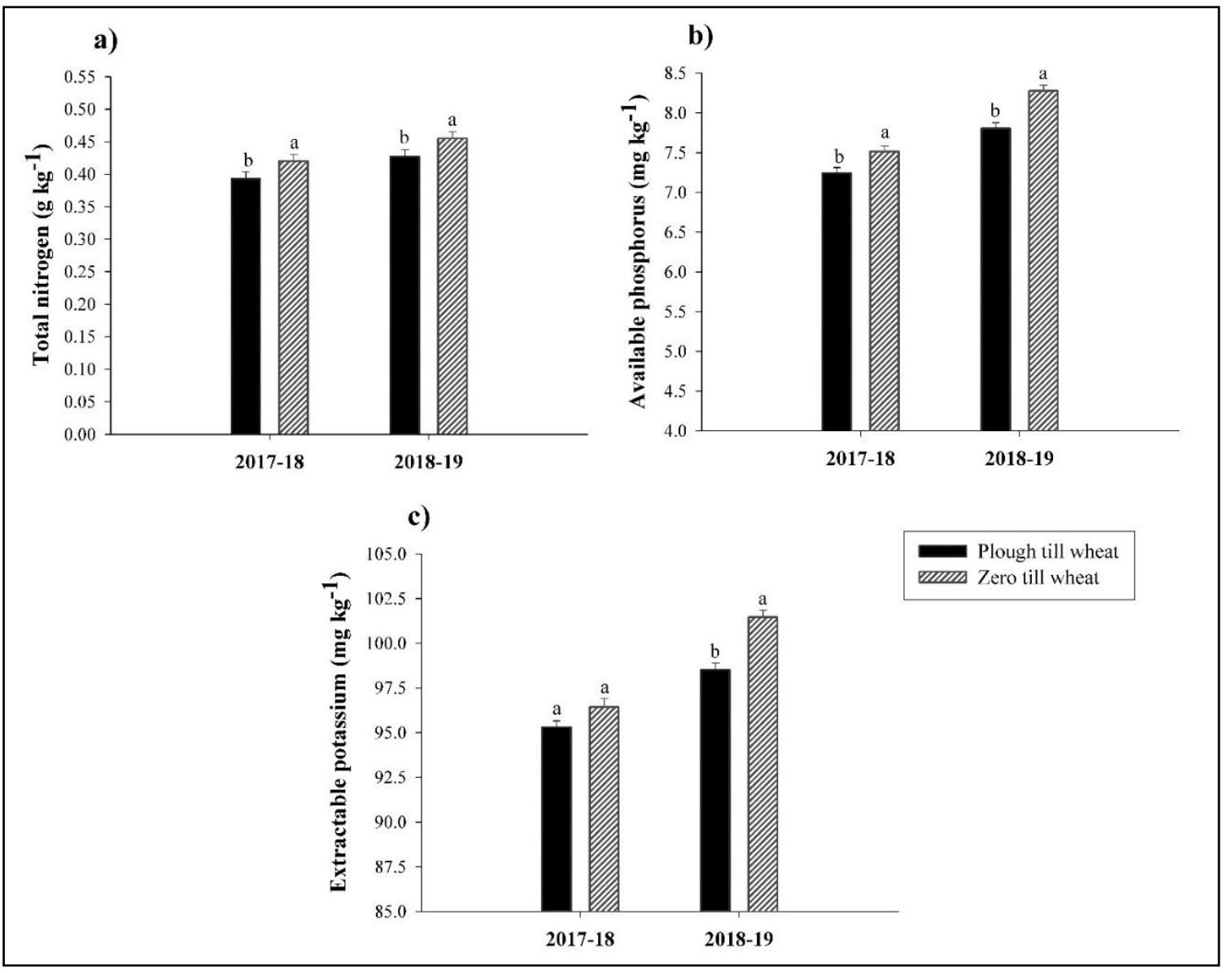

Figure 1. Influence of plough tillage and zero tillage system on (a) total nitrogen $\left(\mathrm{g} \mathrm{kg}^{-1}\right)$, (b) available phosphorous $\left(\mathrm{mg} \mathrm{kg}^{-1}\right)$, and (c) extractable potassium $\left(\mathrm{mg} \mathrm{kg}^{-1}\right)$. Error bars above means indicate the \pm S.E. of three replicates. Means sharing the same letter during an experimental year for a parameter do not differ significantly at $p \leq 0.05$.

\subsection{Yield Attributes}

Application of $\mathrm{Zn}$ significantly influenced the number of productive tillers during both years; however, the effect of tillage systems was non-significant for productive tillers. Averaged across different wheat tillage systems (WTs), the number of productive tillers was $14.08 \%$ and $12.44 \%$ higher with Zn priming during 2017-2018 and 2018-2019, respectively, compared with control. Grains per spike were considerably influenced by tillage systems and Zn nutrition; ZT produced higher number of grains per spike in comparison to PT and among Zn application methods, 38.2\% higher number 
of grains per spike were found with Zn priming in comparison to control treatment during the first study year. For the second experimental year, the highest grains per spike (34.28\% over control) were found in response to Zn seed priming under ZT system. Tillage system had no significant impact on 1000-grain weight, whereas $\mathrm{Zn}$ application substantially influenced the grain weight and $\mathrm{Zn}$ seed priming resulted in $26.46 \%$ and $23.45 \%$ increase in 1000 -grain weight relative to control during 2017-2018 and 2018-2019, respectively. Foliar-applied Zn during the second year gave statistically similar results to seed $\mathrm{Zn}$ priming (Table 3 ). The highest biological yield (18.58\% relative to control) was documented through soil application of $\mathrm{Zn}$ that was statistically at par to $\mathrm{Zn}$ seed priming during the first year. However, the interaction of WTs $\times$ Zn was significant during second year and the highest biological yield $(27.40 \%)$ was obtained with soil-applied Zn in PT system. Similarly, the highest grain yield (42.1\% over control) was achieved with Zn seed priming in the ZT system in first year that was statistically at par with Zn seed priming in the PT system. However, the interaction of WTs $\times$ Zn was non-significant during the second year and the highest grain yield ( $32.8 \%$ over control) was obtained with $\mathrm{Zn}$ seed priming. For tillage systems, the higher grain yield was recorded under ZT system in comparison to PT. The highest harvest index was observed in response to $\mathrm{Zn}$ seed priming for both years (Table 4).

Table 3. Effect of $\mathrm{Zn}$ application on yield and related traits of wheat grown under two tillage systems.

\begin{tabular}{|c|c|c|c|c|c|c|}
\hline \multirow{2}{*}{ Table } & \multicolumn{3}{|c|}{$2017-2018$} & \multicolumn{3}{|c|}{ 2018-2019 } \\
\hline & PTW & ZTW & Mean (Zn) & PTW & ZTW & Mean (Zn) \\
\hline \multicolumn{7}{|c|}{ Productive Tillers $\left(\mathrm{m}^{-2}\right)$} \\
\hline No application & $319 d$ & $320 \mathrm{~d}$ & $320 \mathrm{~F}$ & $316 \mathrm{fg}$ & $319 \mathrm{efg}$ & $318 \mathrm{D}$ \\
\hline Zn-coating & $340 \mathrm{~b}$ & $336 \mathrm{bc}$ & $338 \mathrm{C}$ & $335 \mathrm{~cd}$ & $333 \mathrm{~cd}$ & $334 \mathrm{BC}$ \\
\hline Hydro-priming & $325 \mathrm{~cd}$ & $328 \mathrm{~cd}$ & $327 \mathrm{DE}$ & 317 efg & $313 \mathrm{~g}$ & $315 \mathrm{D}$ \\
\hline Zn-priming & $365 a$ & $364 \mathrm{a}$ & $365 \mathrm{~A}$ & $354 \mathrm{ab}$ & $360 \mathrm{a}$ & $357 \mathrm{~A}$ \\
\hline Soil application & $355 a$ & $354 \mathrm{a}$ & $355 \mathrm{~B}$ & 344 bc & $330 \mathrm{de}$ & $337 \mathrm{~B}$ \\
\hline Hydro-foliar & $320 \mathrm{~d}$ & $325 \mathrm{~cd}$ & $323 \mathrm{EF}$ & 317 efg & $315 \mathrm{fg}$ & $316 \mathrm{D}$ \\
\hline Zn-foliar & $327 \mathrm{~cd}$ & 329 bcd & $328 \mathrm{D}$ & 327 def & 322 defg & $325 \mathrm{CD}$ \\
\hline Mean (WTs) & $336 \mathrm{~A}$ & $337 \mathrm{~A}$ & & $330 \mathrm{~A}$ & $327 \mathrm{~A}$ & \\
\hline \multirow{2}{*}{\multicolumn{7}{|c|}{ HSD $(p \leq 0.05)$}} \\
\hline & & & & & & \\
\hline No application & $33 \mathrm{~g}$ & $35 \mathrm{ef}$ & $34 \mathrm{~F}$ & $35 \mathrm{e}$ & $36 \mathrm{e}$ & $36 \mathrm{C}$ \\
\hline Zn-coating & $41 \mathrm{~d}$ & $41 \mathrm{~d}$ & $41 \mathrm{D}$ & $41 \mathrm{~d}$ & $41 \mathrm{~d}$ & $41 \mathrm{C}$ \\
\hline Hydro-priming & $34 \mathrm{fg}$ & $35 \mathrm{ef}$ & $35 \mathrm{EF}$ & $36 \mathrm{e}$ & $36 \mathrm{e}$ & $36 \mathrm{C}$ \\
\hline Zn-priming & $47 \mathrm{a}$ & $47 \mathrm{a}$ & $47 \mathrm{~A}$ & $45 \mathrm{~b}$ & $47 \mathrm{a}$ & $46 \mathrm{~A}$ \\
\hline Soil application & $43 c$ & $44 \mathrm{bc}$ & $44 \mathrm{C}$ & $43 c$ & $43 c$ & $43 \mathrm{~B}$ \\
\hline Hydro-foliar & $35 \mathrm{ef}$ & $36 \mathrm{e}$ & $36 \mathrm{E}$ & $36 \mathrm{e}$ & $35 \mathrm{e}$ & $36 \mathrm{C}$ \\
\hline Zn-foliar & $45 \mathrm{~b}$ & $45 \mathrm{~b}$ & $45 \mathrm{~B}$ & $47 \mathrm{a}$ & $45 \mathrm{~b}$ & $46 \mathrm{~A}$ \\
\hline Mean (WTs) & $39.7 \mathrm{~B}$ & $40.4 \mathrm{~A}$ & & $40.4 \mathrm{~A}$ & $40.4 \mathrm{~A}$ & \\
\hline $\operatorname{HSD}(p \leq 0.05)$ & \multicolumn{3}{|c|}{$\mathrm{WTs}=0.7 ; \mathrm{Zn}=0.61$} & \multicolumn{3}{|c|}{$\mathrm{Zn}=0.93 ; \mathrm{WTs} \times \mathrm{Zn}=1.32$} \\
\hline \multicolumn{7}{|c|}{ 1000-grain Weight (g) } \\
\hline No application & $35.17 \mathrm{f}$ & $35.36 \mathrm{f}$ & $35.26 \mathrm{D}$ & $36.07 \mathrm{~d}$ & $35.97 \mathrm{~d}$ & $36.02 \mathrm{D}$ \\
\hline Zn-coating & 38.00 ef & 38.92 de & $38.46 \mathrm{C}$ & $38.37 \mathrm{c}$ & $38.35 \mathrm{c}$ & $38.36 \mathrm{C}$ \\
\hline Hydro-priming & $35.97 \mathrm{f}$ & $35.78 \mathrm{f}$ & $35.87 \mathrm{D}$ & $35.97 \mathrm{~d}$ & $36.84 \mathrm{~d}$ & $36.41 \mathrm{D}$ \\
\hline Zn-priming & $44.89 \mathrm{a}$ & $44.30 \mathrm{ab}$ & $44.59 \mathrm{~A}$ & $43.98 \mathrm{a}$ & $44.97 \mathrm{a}$ & $44.47 \mathrm{~A}$ \\
\hline Soil application & $41.16 \mathrm{~cd}$ & $41.83 \mathrm{bcd}$ & $41.49 \mathrm{~B}$ & $41.16 \mathrm{~b}$ & $42.36 \mathrm{~b}$ & $41.76 \mathrm{~B}$ \\
\hline Hydro-foliar & $35.15 \mathrm{f}$ & $36.32 \mathrm{ef}$ & $35.73 \mathrm{D}$ & $36.15 \mathrm{~d}$ & $36.12 \mathrm{~d}$ & $36.13 \mathrm{D}$ \\
\hline Zn-foliar & $42.70 \mathrm{abc}$ & $42.24 \mathrm{abc}$ & $42.47 \mathrm{~B}$ & $44.04 \mathrm{a}$ & $44.41 \mathrm{a}$ & $44.22 \mathrm{~A}$ \\
\hline Mean (WTs) & $39.00 \mathrm{~A}$ & $39.25 \mathrm{~A}$ & & $39.39 \mathrm{~A}$ & $39.86 \mathrm{~A}$ & \\
\hline $\operatorname{HSD}(p \leq 0.05)$ & & $\mathrm{Zn}=1.68$ & & & $\mathrm{Zn}=1.24$ & \\
\hline
\end{tabular}

Means sharing the same uppercase and lowercase letter for main effects and interaction do not differ significantly at $(p \leq 0.05)$ for a parameter during growing season by Tukey's honestly significant difference (HSD) test; PTW = Plough till wheat; ZTW = Zero till wheat; WTs = Wheat tillage systems. 
Table 4. Effect of Zn application on yield and related traits of wheat grown under two tillage systems.

\begin{tabular}{|c|c|c|c|c|c|c|}
\hline \multirow{2}{*}{ Treatments } & \multicolumn{3}{|c|}{ 2017-2018 } & \multicolumn{3}{|c|}{ 2018-2019 } \\
\hline & PTW & ZTW & Mean (Zn) & PTW & ZTW & Mean (Zn) \\
\hline \multicolumn{7}{|c|}{ Biological Yield (Mg ha $\left.{ }^{-1}\right)$} \\
\hline No application & $7.39 \mathrm{~g}$ & $7.57 \mathrm{f}$ & $7.48 \mathrm{E}$ & $6.93 \mathrm{j}$ & $6.86 \mathrm{j}$ & $6.89 \mathrm{G}$ \\
\hline Zn-coating & $8.11 \mathrm{de}$ & 7.98 ef & $8.04 \mathrm{C}$ & $7.79 \mathrm{e}$ & $7.51 \mathrm{f}$ & $7.65 \mathrm{D}$ \\
\hline Hydro-priming & $7.77 \mathrm{fgi}$ & $7.78 \mathrm{fg}$ & $7.77 \mathrm{D}$ & $7.41 \mathrm{fg}$ & $7.32 \mathrm{gh}$ & $7.36 \mathrm{E}$ \\
\hline Zn-priming & $8.77 \mathrm{ab}$ & $8.81 \mathrm{a}$ & $8.79 \mathrm{~A}$ & $8.49 \mathrm{~b}$ & $8.21 \mathrm{c}$ & $8.35 \mathrm{~B}$ \\
\hline Soil application & $8.86 \mathrm{a}$ & $8.88 \mathrm{a}$ & $8.87 \mathrm{~A}$ & $8.74 \mathrm{a}$ & $8.31 \mathrm{bc}$ & $8.52 \mathrm{~A}$ \\
\hline Hydro-foliar & $7.45 \mathrm{fg}$ & $7.60 \mathrm{ef}$ & $7.52 \mathrm{E}$ & $7.19 \mathrm{hi}$ & $7.15 \mathrm{i}$ & $7.17 \mathrm{~F}$ \\
\hline Zn-foliar & $8.49 \mathrm{~b}$ & $8.35 \mathrm{~b}$ & $8.42 \mathrm{~B}$ & $8.29 c$ & $7.98 \mathrm{~d}$ & $8.14 \mathrm{C}$ \\
\hline Mean (WTs) & $8.12 \mathrm{~A}$ & $8.14 \mathrm{~A}$ & & $7.83 \mathrm{~A}$ & $7.62 \mathrm{~A}$ & \\
\hline $\operatorname{HSD}(p \leq 0.05)$ & \multicolumn{3}{|c|}{$\mathrm{Zn}=0.12$} & \multicolumn{3}{|c|}{$\mathrm{Zn}=0.08 ; \mathrm{WTs} \times \mathrm{Zn}=0.12$} \\
\hline \multicolumn{7}{|c|}{ Grain Yield $\left(\mathrm{Mg} \mathrm{ha}^{-1}\right)$} \\
\hline No application & $3.13 \mathrm{f}$ & $3.26 \mathrm{f}$ & $3.19 \mathrm{~F}$ & $3.23 \mathrm{hi}$ & $3.35 \mathrm{fg}$ & $3.29 \mathrm{E}$ \\
\hline Zn-coating & $3.85 \mathrm{c}$ & $3.87 \mathrm{c}$ & $3.86 \mathrm{C}$ & $3.53 \mathrm{e}$ & $3.64 \mathrm{~d}$ & $3.59 \mathrm{D}$ \\
\hline Hydro-priming & 3.26 ef & $3.40 \mathrm{e}$ & $3.33 \mathrm{E}$ & $3.18 \mathrm{i}$ & $3.37 \mathrm{f}$ & $3.28 \mathrm{E}$ \\
\hline Zn-priming & $4.42 \mathrm{a}$ & $4.45 \mathrm{a}$ & $4.43 \mathrm{~A}$ & $4.35 \mathrm{a}$ & $4.39 \mathrm{a}$ & $4.37 \mathrm{~A}$ \\
\hline Soil application & $4.13 \mathrm{~b}$ & $4.15 \mathrm{~b}$ & $4.14 \mathrm{~B}$ & $3.98 \mathrm{~b}$ & $4.10 \mathrm{~b}$ & $4.06 \mathrm{~B}$ \\
\hline Hydro-foliar & $3.22 \mathrm{f}$ & $3.23 \mathrm{f}$ & $3.22 \mathrm{~F}$ & $3.28 \mathrm{gh}$ & $3.32 \mathrm{fgh}$ & $3.30 \mathrm{E}$ \\
\hline Zn-foliar & $3.58 \mathrm{~d}$ & $3.80 \mathrm{c}$ & $3.69 \mathrm{D}$ & $3.76 \mathrm{c}$ & $3.62 \mathrm{~d}$ & $3.69 \mathrm{C}$ \\
\hline Mean (WTs) & $3.65 \mathrm{~B}$ & $3.74 \mathrm{~A}$ & & $3.58 \mathrm{~B}$ & $3.64 \mathrm{~A}$ & \\
\hline $\operatorname{HSD}(p \leq 0.05)$ & \multicolumn{3}{|c|}{$\mathrm{WTs}=0.015 ; \mathrm{Zn}=0.09 ; \mathrm{WTs} \times \mathrm{Zn}=0.15$} & \multicolumn{3}{|c|}{$\mathrm{WTs}=0.03 ; \mathrm{Zn}=0.097$} \\
\hline \multicolumn{7}{|c|}{ Harvest Index (\%) } \\
\hline No application & $42.36 \mathrm{ef}$ & 43.05 ef & $42.71 \mathrm{E}$ & $46.71 \mathrm{a}$ & $48.84 \mathrm{a}$ & $47.77 \mathrm{~B}$ \\
\hline Zn-coating & $47.47 \mathrm{bc}$ & $48.50 \mathrm{~b}$ & $47.98 \mathrm{~B}$ & $45.32 \mathrm{a}$ & $48.47 \mathrm{a}$ & $46.90 \mathrm{BC}$ \\
\hline Hydro-priming & $41.96 \mathrm{f}$ & $43.70 \mathrm{e}$ & $42.83 \mathrm{DE}$ & $42.91 \mathrm{a}$ & $46.04 \mathrm{a}$ & $44.47 \mathrm{D}$ \\
\hline Zn-priming & $50.40 \mathrm{a}$ & $50.51 \mathrm{a}$ & $50.45 \mathrm{~A}$ & $51.24 \mathrm{a}$ & $53.47 \mathrm{a}$ & $52.35 \mathrm{~A}$ \\
\hline Soil application & $46.61 \mathrm{~cd}$ & $46.73 \mathrm{bcd}$ & $46.67 \mathrm{C}$ & $45.99 \mathrm{a}$ & $49.33 \mathrm{a}$ & $47.66 \mathrm{~B}$ \\
\hline Hydro-foliar & 43.22 ef & 42.50 ef & $42.86 \mathrm{DE}$ & $45.62 \mathrm{a}$ & $46.44 \mathrm{a}$ & $46.03 \mathrm{BCD}$ \\
\hline Zn-foliar & $42.16 \mathrm{ef}$ & $45.51 \mathrm{~d}$ & $43.83 \mathrm{D}$ & $45.36 \mathrm{a}$ & $45.45 \mathrm{a}$ & $45.40 \mathrm{CD}$ \\
\hline Mean (WTs) & $44.9 \mathrm{~A}$ & $45.8 \mathrm{~A}$ & & $46.16 \mathrm{~B}$ & $48.29 \mathrm{~A}$ & \\
\hline $\operatorname{HSD}(p \leq 0.05)$ & \multicolumn{3}{|c|}{$\mathrm{Zn}=1.09 ; \mathrm{WTs} \times \mathrm{Zn}=1.78$} & \multicolumn{3}{|c|}{$\mathrm{WTs}=1.30 ; \mathrm{Zn}=1.75$} \\
\hline
\end{tabular}

Means sharing the same uppercase and lowercase letter for main effects and interaction do not differ significantly at $(p \leq 0.05)$ for a parameter during growing season by Tukey's honestly significant difference (HSD) test; PTW = Plough till wheat; ZTW = Zero till wheat; WTs = Wheat tillage systems.

\subsection{Grain and Straw Zn Concentration}

Grain and straw $\mathrm{Zn}$ concentration were significantly influenced by $\mathrm{Zn}$ application methods. In the PT system, the highest grain Zn contents were noted with foliar-applied Zn during both experimental years, whereas in the ZT system, soil-applied $\mathrm{Zn}$ resulted in the highest grain $\mathrm{Zn}$ concentration, whereas the lowest grain $\mathrm{Zn}$ concentration was observed in no $\mathrm{Zn}$ application, followed by hydro-priming and foliar water spray in both tillage systems (Figure 2a,b). The highest straw Zn contents were observed with soil-applied Zn in PT as well as ZT system and this treatment was followed by foliar application of $\mathrm{Zn}$ during both years (Figure 2c,d).

\subsection{Bioavailable Zn Contents in Bread Wheat Grains}

Zinc application through different methods significantly influenced the bioavailable $\mathrm{Zn}$ contents during both study years. Foliar application of $\mathrm{Zn}$ improved the bioavailability of $\mathrm{Zn}$ while it reduced the grain phytate contents and [phytate]:[Zn] during both study years. However, for the second year, foliar application of $\mathrm{Zn}$ recorded statistically similar effects compared to soil application of $\mathrm{Zn}$ (Table 5). 


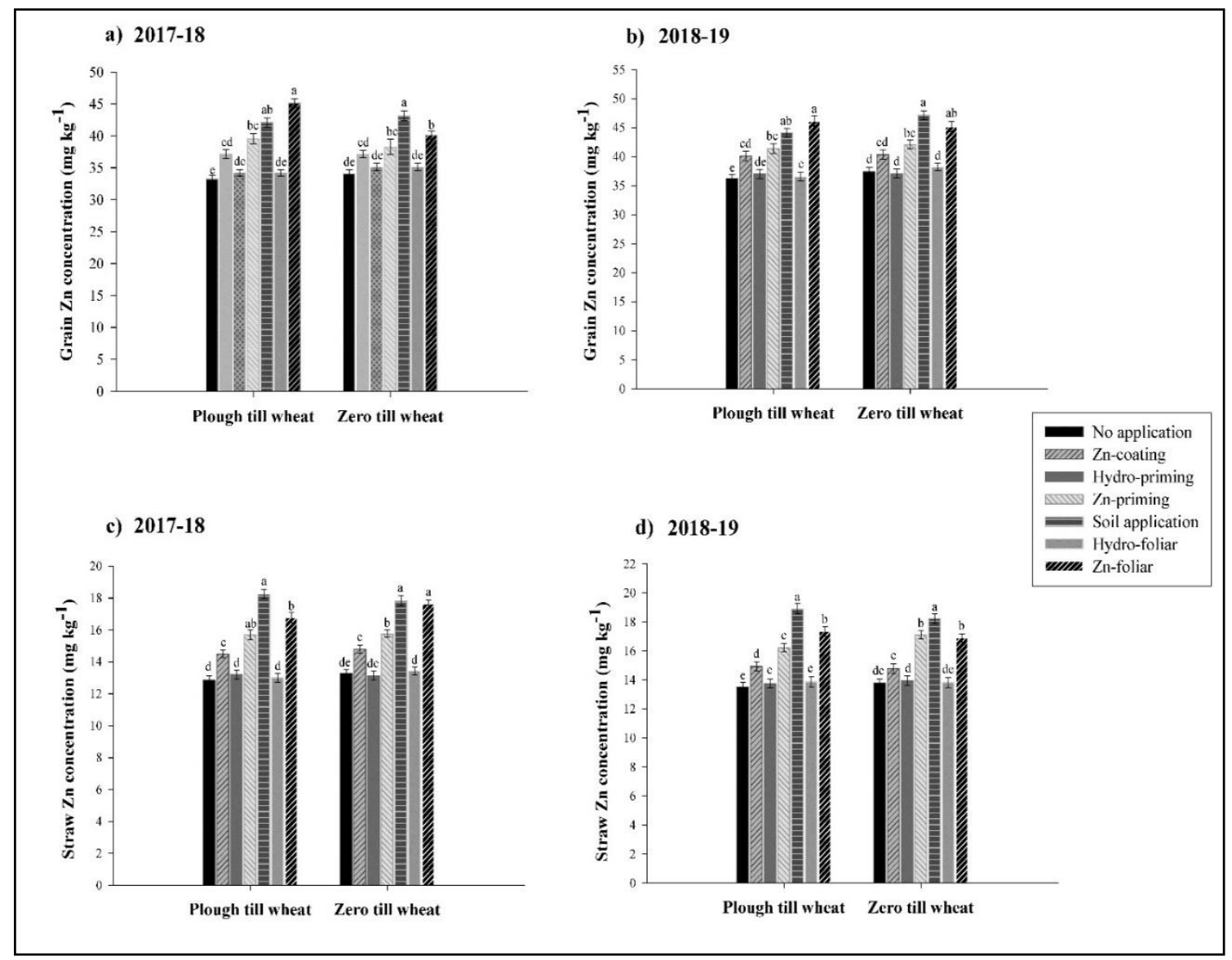

Figure 2. Influence of $\mathrm{Zn}$ application on grain $\mathrm{Zn}$ concentration $\left(\mathrm{mg} \mathrm{kg}^{-1}\right)$ during (a) 2017-2018 and (b) 2018-2019 and straw Zn concentration ( $\mathrm{mg} \mathrm{kg}^{-1}$ ) of wheat during (c) 2017-2018 and (d) 2018-2019. Error bars above means indicate the \pm S.E. of three replicates. Means sharing the same letter during an experimental year for a parameter do not differ significantly at $p \leq 0.05$.

Table 5. Effect of different $\mathrm{Zn}$ application methods on grain phytate, phytate into $\mathrm{Zn}$ molar ratio, and bioavailable $\mathrm{Zn}$ content under two tillage systems.

\begin{tabular}{|c|c|c|c|c|c|c|}
\hline \multirow{2}{*}{ Treatments } & \multicolumn{3}{|c|}{ 2017-2018 } & \multicolumn{3}{|c|}{ 2018-2019 } \\
\hline & PTW & ZTW & Mean (Zn) & PTW & ZTW & Mean (Zn) \\
\hline \multicolumn{7}{|c|}{ Phytic Acid (mg g $\left.{ }^{-1}\right)$} \\
\hline No application & $12.46 \mathrm{a}$ & $12.39 \mathrm{a}$ & $12.42 \mathrm{~A}$ & $13.05 \mathrm{ab}$ & $13.13 \mathrm{a}$ & $13.09 \mathrm{~A}$ \\
\hline Zn-coating & $11.06 \mathrm{a}$ & $11.39 \mathrm{a}$ & $11.22 \mathrm{~B}$ & $12.10 \mathrm{~cd}$ & $12.48 \mathrm{bc}$ & $12.29 \mathrm{~B}$ \\
\hline Hydro-priming & $12.49 \mathrm{a}$ & $12.53 \mathrm{a}$ & $12.51 \mathrm{~A}$ & $13.08 \mathrm{ab}$ & $13.02 \mathrm{ab}$ & $13.05 \mathrm{~A}$ \\
\hline Zn-priming & $10.71 \mathrm{a}$ & $10.85 \mathrm{a}$ & $10.78 \mathrm{C}$ & $11.60 \mathrm{de}$ & $11.74 \mathrm{de}$ & $11.67 \mathrm{C}$ \\
\hline Soil application & $9.89 \mathrm{a}$ & $10.32 \mathrm{a}$ & $10.10 \mathrm{D}$ & $10.78 \mathrm{fg}$ & $11.21 \mathrm{ef}$ & $10.99 \mathrm{D}$ \\
\hline Hydro-foliar & $12.44 \mathrm{a}$ & $12.46 \mathrm{a}$ & $12.45 \mathrm{~A}$ & $13.03 \mathrm{ab}$ & $13.13 \mathrm{a}$ & $13.08 \mathrm{~A}$ \\
\hline Zn-foliar & $9.98 \mathrm{a}$ & $9.62 \mathrm{a}$ & $9.80 \mathrm{D}$ & $10.87 \mathrm{fg}$ & $10.51 \mathrm{~g}$ & $10.69 \mathrm{D}$ \\
\hline Mean (WTs) & $11.29 \mathrm{~A}$ & $11.36 \mathrm{~A}$ & & $12.07 \mathrm{~A}$ & $12.17 \mathrm{~A}$ & \\
\hline $\operatorname{HSD}(p \leq 0.05)$ & \multicolumn{3}{|c|}{$\mathrm{Zn}=0.32$} & \multicolumn{3}{|c|}{$\mathrm{Zn}=0.35 ; \mathrm{WTs} \times \mathrm{Zn}=0.56$} \\
\hline
\end{tabular}


Table 5. Cont.

\begin{tabular}{|c|c|c|c|c|c|c|}
\hline \multicolumn{7}{|c|}{ [Phytate]:[Zn] } \\
\hline No application & $37.13 \mathrm{a}$ & $35.99 \mathrm{ab}$ & $36.56 \mathrm{~A}$ & $35.68 \mathrm{a}$ & $34.78 \mathrm{a}$ & $35.23 \mathrm{~A}$ \\
\hline Zn-coating & $29.50 \mathrm{~cd}$ & $30.40 \mathrm{c}$ & 29.95 B & $29.92 \mathrm{a}$ & $30.66 \mathrm{a}$ & $30.29 \mathrm{~B}$ \\
\hline Hydro-priming & $36.17 \mathrm{ab}$ & $35.37 \mathrm{ab}$ & $35.77 \mathrm{~A}$ & $34.99 \mathrm{a}$ & $34.76 \mathrm{a}$ & $34.87 \mathrm{~A}$ \\
\hline Zn-priming & $26.84 \mathrm{e}$ & $28.10 \mathrm{de}$ & $27.47 \mathrm{C}$ & $27.76 \mathrm{a}$ & $27.64 \mathrm{a}$ & $27.70 \mathrm{C}$ \\
\hline Soil application & $23.28 \mathrm{f}$ & $23.68 \mathrm{f}$ & $23.48 \mathrm{D}$ & $24.21 \mathrm{a}$ & $23.57 \mathrm{a}$ & $23.89 \mathrm{D}$ \\
\hline Hydro-foliar & $36.03 \mathrm{ab}$ & $35.15 \mathrm{~b}$ & $35.59 \mathrm{~A}$ & $35.33 \mathrm{a}$ & $34.04 \mathrm{a}$ & $34.68 \mathrm{~A}$ \\
\hline Zn-foliar & $21.89 \mathrm{f}$ & $23.78 \mathrm{f}$ & $22.83 \mathrm{D}$ & $23.34 \mathrm{a}$ & $23.05 \mathrm{a}$ & $23.19 \mathrm{D}$ \\
\hline Mean (WTs) & $30.12 \mathrm{~A}$ & $30.35 \mathrm{~A}$ & & $30.17 \mathrm{~A}$ & $29.78 \mathrm{~A}$ & \\
\hline $\operatorname{HSD}(p \leq 0.05)$ & \multicolumn{3}{|c|}{$\mathrm{Zn}=1.49 ; \mathrm{WTs} \times \mathrm{Zn}=1.49$} & & $\mathrm{Zn}=1.25$ & \\
\hline \multicolumn{7}{|c|}{ Estimated Bioavailable Zinc (mg $300 \mathrm{~g}^{-1}$ flour) } \\
\hline No application & $1.99 \mathrm{e}$ & $2.03 \mathrm{e}$ & $2.01 \mathrm{E}$ & $2.05 \mathrm{f}$ & $2.09 \mathrm{ef}$ & $2.07 \mathrm{D}$ \\
\hline Zn-coating & $2.30 \mathrm{c}$ & $2.26 \mathrm{~cd}$ & $2.28 \mathrm{CD}$ & $2.29 \mathrm{~cd}$ & $2.26 \mathrm{cde}$ & $2.27 \mathrm{C}$ \\
\hline Hydro-priming & $2.03 \mathrm{e}$ & $2.06 \mathrm{de}$ & $2.04 \mathrm{DE}$ & $2.08 \mathrm{ef}$ & 2.09 ef & $2.08 \mathrm{D}$ \\
\hline Zn-priming & $2.43 \mathrm{bc}$ & $2.37 \mathrm{c}$ & $2.40 \mathrm{BC}$ & $2.39 c$ & $2.40 \mathrm{bc}$ & $2.39 \mathrm{~B}$ \\
\hline Soil application & $2.63 \mathrm{ab}$ & $2.61 \mathrm{ab}$ & $2.62 \mathrm{AB}$ & $2.59 \mathrm{ab}$ & $2.63 \mathrm{a}$ & $2.61 \mathrm{~A}$ \\
\hline Hydro-foliar & $2.03 \mathrm{e}$ & $2.07 \mathrm{de}$ & $2.05 \mathrm{DE}$ & 2.07 ef & $2.12 \mathrm{def}$ & $2.09 \mathrm{D}$ \\
\hline Zn-foliar & $2.73 \mathrm{a}$ & $2.60 \mathrm{ab}$ & $2.66 \mathrm{~A}$ & $2.64 \mathrm{a}$ & $2.66 \mathrm{a}$ & $2.65 \mathrm{~A}$ \\
\hline Mean (WTs) & $2.30 \mathrm{~A}$ & $2.28 \mathrm{~A}$ & & $2.30 \mathrm{~A}$ & $2.32 \mathrm{~A}$ & \\
\hline $\operatorname{HSD}(p \leq 0.05)$ & & $\mathrm{Zn}=0.23$ & & & $\mathrm{Zn}=0.112$ & \\
\hline
\end{tabular}

Means sharing the same uppercase and lowercase letter for main effects and interaction do not differ significantly at $(p \leq 0.05)$ for a parameter during growing season by Tukey's honestly significant difference (HSD) test; PTW = Plough till wheat; ZTW = Zero till wheat; WTs = Wheat tillage systems.

\subsection{Zinc Use Efficiency Indices}

Zinc application methods substantially affected the efficiency indices during the first and second year (Table 6), whereas the ARE during the first year was considerably affected by WTs (Table 6). Higher AgE was observed with Zn-coating under PT during both the years. The highest PE was noted with $\mathrm{Zn}$ seed priming for first year of study, whereas results were non-significant during the second year. Agro-physiological efficiency (AgPE) was the highest with Zn seed priming during the second year; however, the results of the AgPE for the first year were non-significant. The ARE was the highest with Zn seed coating during 2017-2018 and 2018-2019, and these results were statistically similar with foliar-applied Zn. Similarly, the highest UE was observed with Zn seed coating during the first year. The interaction of WTs $\times$ Zn was significant during the second year and the highest UE was observed when $\mathrm{Zn}$ coated wheat seeds were sown under PT system. The interactive effect of WTs $\times \mathrm{Zn}$ for PFP was significant and the highest PFP was noted with Zn seed coating and ZT during both years (Table 6). 
Table 6. Effect of $\mathrm{Zn}$ application methods on $\mathrm{Zn}$ use efficiencies of wheat under two tillage systems.

\begin{tabular}{|c|c|c|c|c|c|c|}
\hline \multirow{2}{*}{ Treatments } & \multicolumn{3}{|c|}{ 2017-2018 } & \multicolumn{3}{|c|}{ 2018-2019 } \\
\hline & PTW & ZTW & Mean (Zn) & PTW & ZTW & Mean (Zn) \\
\hline \multicolumn{7}{|c|}{ Agronomic Efficiency $\left(\mathrm{kg} \mathrm{kg}^{-1}\right)$} \\
\hline Zn-coating & $4615.4 \mathrm{a}$ & $3910.3 \mathrm{~b}$ & $4262.8 \mathrm{~A}$ & $1880.3 \mathrm{a}$ & $1859.0 \mathrm{a}$ & $1869.7 \mathrm{~A}$ \\
\hline Zn-priming & $970.00 \mathrm{c}$ & 900.00 c & $935.00 \mathrm{~B}$ & $750.00 \mathrm{bc}$ & $750.00 \mathrm{bc}$ & $750.00 \mathrm{~B}$ \\
\hline Soil application & $108.00 \mathrm{~d}$ & $96.000 \mathrm{~d}$ & $102.00 \mathrm{C}$ & $91.300 \mathrm{c}$ & $75.000 \mathrm{c}$ & $83.200 \mathrm{C}$ \\
\hline Zn-Foliar & $937.50 \mathrm{c}$ & $1125.0 \mathrm{c}$ & 1031.2 B & $1090.3 \mathrm{ab}$ & $576.40 \mathrm{bc}$ & $833.30 \mathrm{~B}$ \\
\hline Mean (WTs) & $1657.7 \mathrm{~A}$ & $1507.8 \mathrm{~A}$ & & $952.99 \mathrm{~A}$ & $815.09 \mathrm{~A}$ & \\
\hline $\operatorname{HSD}(p \leq 0.05)$ & \multicolumn{3}{|c|}{$\mathrm{Zn}=233.0 ; \mathrm{WTs} \times \mathrm{Zn}=241.7$} & \multicolumn{3}{|c|}{$\mathrm{Zn}=501.90 ; \mathrm{WTs} \times \mathrm{Zn}=775.21$} \\
\hline \multicolumn{7}{|c|}{ Physiological efficiency $\left(\mathrm{kg} \mathrm{kg}^{-1}\right)$} \\
\hline Zn-coating & $130.85 \mathrm{a}$ & $95.810 \mathrm{a}$ & $113.33 \mathrm{~B}$ & $170.28 \mathrm{a}$ & $242.15 \mathrm{a}$ & $206.21 \mathrm{~A}$ \\
\hline Zn-priming & $163.75 \mathrm{a}$ & $198.22 \mathrm{a}$ & $180.98 \mathrm{~A}$ & $202.32 \mathrm{a}$ & $173.76 \mathrm{a}$ & $188.04 \mathrm{~A}$ \\
\hline Soil application & $122.48 \mathrm{a}$ & $104.70 \mathrm{a}$ & $113.59 \mathrm{~B}$ & $152.26 \mathrm{a}$ & $118.98 \mathrm{a}$ & $135.62 \mathrm{~A}$ \\
\hline Zn-Foliar & $70.140 \mathrm{a}$ & $76.220 \mathrm{a}$ & $73.18 \mathrm{C}$ & $99.750 \mathrm{a}$ & $106.25 \mathrm{a}$ & $103.00 \mathrm{~A}$ \\
\hline Mean (WTs) & $121.81 \mathrm{~A}$ & $118.74 \mathrm{~A}$ & & $156.15 \mathrm{~A}$ & $160.28 \mathrm{~A}$ & \\
\hline \multirow{2}{*}{\multicolumn{7}{|c|}{ HSD $(p \leq 0.05)$}} \\
\hline & & & & & & \\
\hline Zn-coating & $133.4 \mathrm{a}$ & $151.6 \mathrm{a}$ & $142.5 \mathrm{~A}$ & $54.80 \mathrm{abc}$ & $95.05 \mathrm{ab}$ & $74.92 \mathrm{AB}$ \\
\hline Zn-priming & $107.2 \mathrm{a}$ & $146.1 \mathrm{a}$ & $126.6 \mathrm{~A}$ & $96.42 \mathrm{ab}$ & $98.04 \mathrm{a}$ & $97.23 \mathrm{~A}$ \\
\hline Soil application & $75.83 \mathrm{a}$ & $70.78 \mathrm{a}$ & $73.31 \mathrm{~A}$ & $69.50 \mathrm{abc}$ & $53.95 \mathrm{bc}$ & $61.72 \mathrm{BC}$ \\
\hline Zn-Foliar & $28.89 \mathrm{a}$ & $52.88 \mathrm{a}$ & $40.89 \mathrm{~A}$ & $38.48 \mathrm{c}$ & $25.34 \mathrm{c}$ & $31.91 \mathrm{C}$ \\
\hline Mean (WTs) & $86.33 \mathrm{~A}$ & $105.33 \mathrm{~A}$ & & $64.80 \mathrm{~A}$ & $68.09 \mathrm{a}$ & \\
\hline $\operatorname{HSD}(p \leq 0.05)$ & & & & & $\mathrm{Zn}=30$ & \\
\hline \multicolumn{7}{|c|}{ Apparent Recovery Efficiency (\%) } \\
\hline Zn-coating & $35.55 \mathrm{a}$ & $29.03 \mathrm{ab}$ & $32.29 \mathrm{~A}$ & $33.61 \mathrm{a}$ & $24.96 \mathrm{a}$ & $29.28 \mathrm{~A}$ \\
\hline Zn-priming & $9.120 \mathrm{c}$ & $6.650 \mathrm{c}$ & $7.890 \mathrm{~B}$ & $7.870 \mathrm{a}$ & $7.990 \mathrm{a}$ & $7.930 \mathrm{~B}$ \\
\hline Soil application & $1.420 \mathrm{c}$ & $1.350 \mathrm{c}$ & $1.390 \mathrm{~B}$ & $1.320 \mathrm{a}$ & $1.410 \mathrm{a}$ & $1.260 \mathrm{~B}$ \\
\hline Zn-Foliar & $32.96 \mathrm{a}$ & $21.53 \mathrm{~b}$ & $27.25 \mathrm{~A}$ & $28.59 \mathrm{a}$ & $22.64 \mathrm{a}$ & $25.62 \mathrm{~A}$ \\
\hline Mean (WTs) & $19.76 \mathrm{~A}$ & $14.64 \mathrm{~B}$ & & $17.85 \mathrm{~A}$ & $14.25 \mathrm{~A}$ & \\
\hline $\operatorname{HSD}(p \leq 0.05)$ & \multicolumn{3}{|c|}{$\mathrm{WTs}=4.85 ; \mathrm{Zn}=10.11$} & \multicolumn{3}{|c|}{$\mathrm{Zn}=10.44$} \\
\hline \multicolumn{7}{|c|}{ Utilization Efficiency $\left(\mathrm{kg} \mathrm{kg}^{-1}\right)$} \\
\hline Zn-coating & $4615.4 \mathrm{a}$ & $2628.2 \mathrm{a}$ & $3621.8 \mathrm{~A}$ & $5512.8 \mathrm{a}$ & $4166.7 \mathrm{~b}$ & $4839.7 \mathrm{~A}$ \\
\hline Zn-priming & $1480.0 \mathrm{a}$ & $1240.0 \mathrm{a}$ & $1360.0 \mathrm{~B}$ & 1560.0 de & $1350.0 \mathrm{e}$ & $1455.0 \mathrm{C}$ \\
\hline Soil application & $174.00 \mathrm{a}$ & $142.00 \mathrm{a}$ & $158.00 \mathrm{C}$ & $201.00 \mathrm{f}$ & $163.00 \mathrm{f}$ & $182.00 \mathrm{D}$ \\
\hline Zn-Foliar & $2291.7 \mathrm{a}$ & $1625.0 \mathrm{a}$ & $1958.3 \mathrm{~B}$ & $2833.3 \mathrm{c}$ & $2333.3 \mathrm{~cd}$ & $2583.3 \mathrm{~B}$ \\
\hline Mean (WTs) & $2140.3 \mathrm{~A}$ & $1408.8 \mathrm{~A}$ & & $2626.8 \mathrm{~A}$ & $2003.3 \mathrm{~A}$ & \\
\hline $\operatorname{HSD}(p \leq 0.05)$ & & $=739.27$ & & $\mathrm{Zn}=$ & 504.28; WTs & $n=713.59$ \\
\hline \multicolumn{7}{|c|}{ Partial Factor Productivity $\left(\mathrm{kg} \mathrm{kg}^{-1}\right)$} \\
\hline Zn-coating & $20,064 \mathrm{~b}$ & $24,808 \mathrm{a}$ & $22,436 \mathrm{~A}$ & $23,333 \mathrm{~b}$ & $22,628 \mathrm{a}$ & $22,981 \mathrm{~A}$ \\
\hline Zn-priming & $4100.0 \mathrm{e}$ & $4160.0 \mathrm{e}$ & $4130.0 \mathrm{C}$ & $4100.0 \mathrm{~d}$ & $3987.0 \mathrm{~d}$ & $4043.0 \mathrm{C}$ \\
\hline Soil application & $421.00 \mathrm{f}$ & $422.00 \mathrm{f}$ & $422.00 \mathrm{D}$ & $410.00 \mathrm{e}$ & $415.00 \mathrm{e}$ & $412.00 \mathrm{D}$ \\
\hline Zn-Foliar & $7458.0 \mathrm{~d}$ & $7917.0 \mathrm{c}$ & $7688.0 \mathrm{~B}$ & $7556.0 \mathrm{c}$ & $7833.0 \mathrm{c}$ & $7694.0 \mathrm{~B}$ \\
\hline Mean (WTs) & 8010.9 B & $9326.6 \mathrm{~A}$ & & $8849.7 \mathrm{~A}$ & $8715.8 \mathrm{~A}$ & \\
\hline $\operatorname{HSD}(p \leq 0.05)$ & \multicolumn{3}{|c|}{$\mathrm{WTs}=285.48 ; \mathrm{Zn}=139.49 ; \mathrm{WTs} \times \mathrm{Zn}=197.27$} & \multicolumn{3}{|c|}{$\mathrm{WTs}=68.66 ; \mathrm{Zn}=279.5 ; \mathrm{WTs} \times \mathrm{Zn}=481.7$} \\
\hline
\end{tabular}

\subsection{Economic Analysis}

Regardless of application methods, Zn nutrition greatly enhanced the net benefits and BCR under both WTs (Table 7). Between two WTs, the highest net benefits were obtained for ZT system than PT systems. Among Zn application methods, Zn seed priming resulted in the highest net benefits and BCR under both WTs. 
Table 7. Economics of Zn application methods in wheat planted in plough tillage and zero tillage during 2017-2018 and 2018-2019.

\begin{tabular}{|c|c|c|c|c|c|c|c|c|c|c|c|}
\hline & Treatments & $\begin{array}{l}\text { Grain } \\
\text { Yield } \\
\left(\mathrm{t} \mathrm{ha}^{-1}\right)\end{array}$ & $\begin{array}{c}\text { Straw } \\
\text { Yield } \\
\left(\mathrm{t} \mathrm{ha} \mathbf{a}^{-1}\right)\end{array}$ & $\begin{array}{c}\text { Adjusted } \\
\text { Grain } \\
\text { Yield } \\
\left(t h^{-1}\right)\end{array}$ & $\begin{array}{c}\text { Adjusted } \\
\text { Straw } \\
\text { Yield } \\
\left(\mathrm{t} \mathrm{ha} \mathrm{a}^{-1}\right)\end{array}$ & $\begin{array}{l}\text { Gross } \\
\text { Income } \\
\left(\$ \text { ha }^{-1}\right)\end{array}$ & $\begin{array}{c}\text { Total Fixed } \\
\text { Cost } \\
\left(\$ \text { ha }^{-1}\right)\end{array}$ & $\begin{array}{c}\text { Total } \\
\text { Variable } \\
\text { Cost (\$ } \\
\left.\text { ha }^{-1}\right)\end{array}$ & $\begin{array}{l}\text { Total Cost } \\
\left(\$ \text { ha }^{-1}\right)\end{array}$ & $\begin{array}{c}\text { Net } \\
\text { Benefits } \\
\left(\$ \text { ha }^{-1}\right)\end{array}$ & $\begin{array}{c}\text { Benefit } \\
\text { Cost Ratio }\end{array}$ \\
\hline \multicolumn{12}{|c|}{ 2017-2018 } \\
\hline \multirow{7}{*}{ PTW } & No application & 3.13 & 4.26 & 2.82 & 3.83 & 894.97 & 471.55 & 56.25 & 527.80 & 367.17 & 1.70 \\
\hline & Zn-coating & 3.85 & 4.26 & 3.47 & 3.83 & 1059.50 & 471.55 & 82.42 & 553.98 & 505.53 & 1.91 \\
\hline & Hydro-priming & 3.26 & 4.51 & 2.93 & 4.06 & 935.23 & 471.55 & 57.81 & 529.37 & 405.86 & 1.77 \\
\hline & Zn-priming & 4.42 & 4.35 & 3.98 & 3.92 & 1193.55 & 471.55 & 125.39 & 596.95 & 596.61 & 2.00 \\
\hline & Soil application & 4.13 & 4.73 & 3.72 & 4.26 & 1143.32 & 471.55 & 103.13 & 574.68 & 568.64 & 1.99 \\
\hline & Hydro foliar & 3.22 & 4.23 & 2.90 & 3.81 & 914.27 & 471.55 & 57.81 & 529.37 & 384.91 & 1.73 \\
\hline & Zn-foliar & 3.58 & 4.91 & 3.22 & 4.42 & 1025.23 & 471.55 & 83.20 & 554.76 & 470.47 & 1.85 \\
\hline \multirow{7}{*}{ ZTW } & No application & 3.26 & 4.31 & 2.93 & 3.88 & 926.79 & 437.27 & 56.25 & 493.52 & 433.27 & 1.88 \\
\hline & Zn-coating & 3.87 & 4.11 & 3.48 & 3.70 & 1057.75 & 437.27 & 82.42 & 519.69 & 538.06 & 2.04 \\
\hline & Hydro-priming & 3.40 & 4.38 & 3.06 & 3.94 & 961.73 & 437.27 & 57.81 & 495.08 & 466.66 & 1.94 \\
\hline & Zn-priming & 4.45 & 4.36 & 4.01 & 3.92 & 1200.83 & 437.27 & 125.39 & 562.66 & 638.18 & 2.13 \\
\hline & Soil application & 4.15 & 4.73 & 3.74 & 4.26 & 1147.89 & 437.27 & 103.13 & 540.39 & 607.50 & 2.12 \\
\hline & Hydro foliar & 3.23 & 4.37 & 2.91 & 3.93 & 922.46 & 437.27 & 57.81 & 495.08 & 427.39 & 1.86 \\
\hline & Zn-foliar & 3.80 & 4.55 & 3.42 & 4.10 & 1060.31 & 437.27 & 83.20 & 520.47 & 539.84 & 2.04 \\
\hline \multicolumn{12}{|c|}{ 2018-2019 } \\
\hline \multirow{7}{*}{ PTW } & No application & 3.24 & 3.69 & 2.91 & 3.32 & 895.44 & 568.17 & 56.25 & 624.42 & 271.02 & 1.43 \\
\hline & Zn-coating & 3.53 & 4.26 & 3.18 & 3.83 & 986.38 & 568.17 & 82.42 & 650.59 & 335.79 & 1.52 \\
\hline & Hydro-priming & 3.18 & 4.23 & 2.86 & 3.81 & 905.13 & 568.17 & 57.81 & 625.98 & 279.15 & 1.45 \\
\hline & Zn-priming & 4.35 & 4.14 & 3.92 & 3.73 & 1168.70 & 568.17 & 125.39 & 693.56 & 475.14 & 1.69 \\
\hline & Soil application & 4.02 & 4.72 & 3.62 & 4.25 & 1117.76 & 568.17 & 103.13 & 671.30 & 446.46 & 1.67 \\
\hline & Hydro foliar & 3.28 & 3.91 & 2.95 & 3.52 & 914.48 & 568.17 & 57.81 & 625.98 & 288.50 & 1.46 \\
\hline & Zn-foliar & 3.76 & 4.53 & 3.38 & 4.08 & 1050.33 & 568.17 & 83.20 & 651.38 & 398.95 & 1.61 \\
\hline \multirow{7}{*}{ ZTW } & No application & 3.35 & 3.51 & 3.02 & 3.16 & 913.61 & 526.80 & 56.25 & 583.05 & 330.55 & 1.57 \\
\hline & Zn-coating & 3.64 & 3.87 & 3.28 & 3.48 & 995.06 & 526.80 & 82.42 & 609.23 & 385.84 & 1.63 \\
\hline & Hydro-priming & 3.37 & 3.95 & 3.03 & 3.56 & 936.74 & 526.80 & 57.81 & 584.62 & 352.12 & 1.60 \\
\hline & Zn-priming & 4.39 & 3.82 & 3.95 & 3.44 & 1164.34 & 526.80 & 125.39 & 652.20 & 512.14 & 1.79 \\
\hline & Soil application & 4.10 & 4.21 & 3.69 & 3.79 & 1114.52 & 526.80 & 103.13 & 629.93 & 484.59 & 1.77 \\
\hline & Hydro foliar & 3.32 & 3.83 & 2.99 & 3.45 & 920.25 & 526.80 & 57.81 & 584.62 & 335.63 & 1.57 \\
\hline & Zn-foliar & 3.63 & 4.35 & 3.26 & 3.92 & 1012.41 & 526.80 & 83.20 & 610.01 & 402.40 & 1.66 \\
\hline
\end{tabular}

1 = 128 PKR; USD \$ 10.15/40 kg for grain; USD \$ 1.87/40 kg for straw; PTW = Plough tillage wheat; ZTW = Zero till wheat. 


\section{Discussion}

The experiment supported the hypothesis that the $\mathrm{Zn}$ application would enhance the wheat productivity, profitability, and grain $\mathrm{Zn}$ biofortification cultivated under both tillage systems, and the ZT would perform better as compared to the PT system for Zn nutrition. Zinc nutrition through either method effectively improved the yield and related traits, grain $\mathrm{Zn}$ biofortification, and net profitability under the PT and ZT systems. The Zn-induced improvements in wheat yield were related to increased number of productive tillers, grains per spike, and 1000-grain weight (Table 3).

Tillage systems significantly influenced the soil properties and nutrient dynamics during both years. Nevertheless, Zn nutrition did not have any considerable impact on SBD, TSP, PR, SMC, SMBN, SOC, and nutrient dynamics (Table 2; Figure 1). Higher values of TSP, PR, SMBC, SMBN, SOC, total N, available P, and extractable K and lower SBD were recorded in ZT than PT (Table 2; Figure 1). Under ZT, the lower SBD and the higher TSP might be due to improvement in soil pores continuity [57]. Residues retention on soil surface under ZT leads to the formation of stable aggregates that leads to improvement in TSP and reduction in the infiltration capacity of the soil [58,59]. Moreover, increase in stable aggregates is linked with higher soil porosity, as minimum disturbance of soil increases the soil transmission and storage pores, thereby improving the soil pores [60].

Reduced soil manipulation breaks the zone of soil compaction and provides a favorable environment with significant improvement in biological properties of soil including SMBC, SOC, and SMBN [19,61]. Crop residues present on soil surface improved the health of soil because of the increased availability of $C$ for decomposition as less soil disturbance provides organic carbon for soil microbes on a continuous basis and enhances the activity of soil microbes, which thus results in the highest SMBC in a ZT system [62]. Under ZT, improvement in SOC and SMBC were due to the storage of mineralizable $C$ from surface residues, ultimate improvement in soil biological activities, and enhanced activities soil enzymes including phosphatase and urease [63]. Conversely, the lowest SOC was recorded under PT primarily because of intensive tillage decline in microbial activity and substantial organic carbon loss [64]. In addition, intensive tillage leads to the dispersion of soil particles and exacerbation of carbon-rich macropores and loss of the soil organic matter having higher degradability and poor stability results loss of SOC [65]. Under ZT, the presence of crop residues sustains organic matter in the soil after microbial decomposition; thus, higher SMBN, SOC and SMBC leads to better the soil tilth and fertility on sustained basis. Furthermore, ZT reduces the rate of soil organic matter decay, decreases losses of soil carbon and increases SOC, SMBC, and SMBN [66]. Contrarily, intensive tillage under PT exposes organic $\mathrm{C}$ to the environment and decreased diversity of soil biota, microbial biomass, and $\mathrm{C}$ and $\mathrm{N}$ mineralization, which leads to a reduction in SOC, SMBC, and SMBN as witnessed in this study [67]. Total $\mathrm{N}$ concentration was increased in ZT, which might be due to the release of nutrients, particularly $\mathrm{N}$, after decomposition of previous crop residues $[68,69]$. In contrast, under PT, the concentration of total $\mathrm{N}$ in the soil was reduced, which might be attributed to nitrate leaching [70] and $\mathrm{N}$ volatilization [71]. An increase in microbial activity accelerated the mineralization of nutrients, which enhanced the phosphorus concentration [72] and extractable K [73]. Under PT, intensive ploughing and inversion of soil layer shift less fertile layer to top of the soil [60].

Zinc seed priming was more effective treatment in enhancing the yield and profitability under both WTs. Nonetheless, the yield was the highest under ZT in comparison to the PT system (Table 4). Zinc seed priming ensures early and uniform stand establishment due to the initiation of pre-germination metabolic mechanisms [74]. Primed seeds have readily available germination metabolites at planting time [75]. Thus, better germination process results in uniform crop establishment even under sub-optimal conditions [76]. Seed germination requires sufficient quantity of promptly available $\mathrm{Zn}$ to facilitate better root growth as $\mathrm{Zn}$ seed priming enhanced the ratio of diving cells, which led to higher germination rate and improved tillering, and ultimately resulted in higher wheat yields [77,78]. Moreover, seed priming with Zn enhances the stand establishment and crop growth due to early radicle and coleoptile development [79]. Application of $\mathrm{Zn}$ considerably improved the productive tillers, grains number per spike, and grain weight mainly due to the $\mathrm{Zn}$ involvement in 
major metabolic activities including carbohydrate metabolism, chlorophyll synthesis, and ribosomal functioning [80]. However, foliar application of $\mathrm{Zn}$ may not perform best due to application at later stages and absence of $\mathrm{Zn}$ for plants during initial stage of development. On the other hand, soil-applied $\mathrm{Zn}$ may take more time owing to the slower movement towards root. Additionally, $\mathrm{Zn}$ application in soil faces complex interactions that hinder the $\mathrm{Zn}$ uptake by roots [81].

Foliar-applied Zn in PT system and soil-applied Zn in ZT system was superior in improving the grain $\mathrm{Zn}$ accumulation during both years (Figure 2). As the $\mathrm{Zn}$ applied on foliage at the reproductive stage is quickly transported to reproductive structures of plants, which is further accumulated in developing seeds [82]. Foliar-applied $\mathrm{Zn}$ is absorbed readily by the leaf epidermis, remobilized further, and then translocated into the grain via phloem with the help of Zn-regulating transporter proteins [83]. Due to alkaline calcareous nature of experimental soil, Zn applied in soil under PT system may get adsorbed [84]. In this study, under ZT system, the highest Zn accumulation in grains was recorded with soil-applied $\mathrm{Zn}$. In some cases, foliar application of $\mathrm{Zn}$ leads to higher $\mathrm{Zn}$ levels in the shoot that restricts better translocation towards grains than plants getting $\mathrm{Zn}$ through soil application [85].

Foliar and soil applied Zn improved the Zn bioavailability (Table 5). Application of Zn as foliar and basal treatment increased the bioavailable $\mathrm{Zn}$ by reducing the phytate content as phytate decreases the $\mathrm{Zn}$ absorption by the human intestine [86]. In this study, the reduction in phytate concentration was due to changes in $\mathrm{P}$ absorption from soil and translocation within plant [87]. Additionally, the improvements in bioavailable $\mathrm{Zn}$ were due to decrease in phytate to $\mathrm{Zn}$ molar ratio owing to lower phytate and $\mathrm{P}$ contents in grain provided with higher $\mathrm{Zn}$ content. Moreover, $\mathrm{Zn}$ biofortification and bioavailable $\mathrm{Zn}$ were improved by foliar-applied $\mathrm{Zn}$ due to the reduced anti-nutrient content in grain and lower phytate to $\mathrm{Zn}$ molar ratio, which shows the bioavailability of $\mathrm{Zn}$ in grains [88]. Furthermore, the increase in grain $\mathrm{Zn}$ content may enhance the $\mathrm{Zn}$ concentration in starchy endosperm of wheat grains, which improves the $\mathrm{Zn}$ availability as endosperm has a lower concentration of phytate [89].

The most significant factor for farming community is profit maximization and profitability is principally associated with input cost and economic yield. The highest BCR was observed with $\mathrm{Zn}$ seed priming under both tillage systems due to the maximum grain yield consequently ascertained more beneficial on monetary basis. Among tillage systems, the ZT system had higher net benefits because of no seedbed preparation in comparison with the PT system.

\section{Conclusions}

Zero tillage enhanced the soil physical and biological characteristics and nutrient availability as indicated by higher TSP, PR, SMBC, SMBN, SOC, total N, available $\mathrm{P}$, and extractable $\mathrm{K}$, while we recorded lower soil bulk density compared with PT. The application of Zn by either method (seed coating, priming, soil or foliar application) enhanced the productivity, grain Zn concentration, $\mathrm{Zn}$ use efficiencies, and bioavailability in bread wheat. Nevertheless, Zn seed priming was the most cost-effective method in enhancing the grain yield and net benefits under both tillage systems. The interaction of Zn seed priming in the ZT system proved to be most efficient and economical in enhancing the yield and related traits. The maximum grain $\mathrm{Zn}$ content and bioavailability were found with foliar $\mathrm{Zn}$ application followed by soil application.

Supplementary Materials: The following are available online at http://www.mdpi.com/2073-4395/10/10/1566/s1. Table S1: comprising data as well as percentage difference regarding Figure 1.

Author Contributions: Conceptualization, data curation, investigation, methodology, and writing-original draft, U.Z.; conceptualization, project administration, resources, supervision, validation, and writing-review and editing, S.H.; data curation, investigation, methodology, and software, M.I.; formal analysis, software, visualization, writing - review and editing, A.M.; data curation, methodology, software, and writing-original draft, N.A.; data curation, formal analysis, software, and writing—original draft, M.A.; formal analysis, funding acquisition, validation, and writing - review and editing, M.N.A.; funding acquisition, validation, visualization, writing - original draft, and writing-review and editing, P.A. All authors have read and agreed to the published version of the manuscript.

Funding: APC funded through RSP-2020/180. 
Acknowledgments: The authors acknowledge the University of Agriculture Faisalabad for the support during research. Research in MNA and PA lab is supported by Researchers Supporting Project Number (RSP-2020/180), King Saud University, Riyadh, Saudi Arabia.

Conflicts of Interest: The authors declare no conflict of interest.

\section{References}

1. Nadeem, F.; Farooq, M. Application of micronutrients in rice-wheat cropping systems of South Asia: A review. Rice Sci. 2019, 26, 356-371. [CrossRef]

2. Erdal, I.; Yilmaz, A.; Taban, S.; Eker, S.; Torun, B.; Cakmak, I. Phytic acid and phosphorus concentrations in seeds of wheat cultivars grown with and without zinc fertilization. J. Plant Nutr. 2002, 25, 113-127. [CrossRef]

3. Cakmak, I. Enrichment of cereal grains with zinc: Agronomic or genetic biofortification? Plant Soil. 2008, 302, 1-17. [CrossRef]

4. Shewry, P.R. Wheat. J. Exp. Bot. 2009, 60, 1537-1553. [CrossRef] [PubMed]

5. Cakmak, I. Zinc Plays Critical Role in Plant Growth. 2011. Available online: http://www.zinc.org/crops/ resourceserve/zinc_plays_critical_role_in_plant_growth (accessed on 17 April 2011).

6. Hussain, S.; Maqsood, M.A.; Rahmatullah. Zinc release characteristics from calcareous soils using di-ethylenetri-aminepentaacetic acid and other organic acids. Commun. Soil Sci. Plant Anal. 2011, 42, 1870-1881. [CrossRef]

7. Alloway, B.J. Soil factors associated with zinc deficiency in crops and humans. Environ. Geochem. Health 2009, 31, 537-548. [CrossRef]

8. Kumssa, D.B.; Joy, E.J.M.; Ander, E.L.; Watts, M.J.L.; Young, S.D.; Rosanoff, A.; White, P.J.; Walker, S.; Broadley, M.R. Global magnesium supply in the food chain. Crop Pasture Sci. 2015, 66, 1278-1289. [CrossRef]

9. Kumssa, D.B.; Joy, E.J.; Ander, E.L.; Watts, M.J.; Young, S.D.; Walker, S.; Broadley, M.R. Dietary calcium and zinc deficiency risks are decreasing but remain prevalent. Sci. Rep. 2015, 5, 10974. [CrossRef]

10. Ministry of Health. National health policy 2009. In Stepping Towards Better Health; Ministry of Health: Islamabad, Pakistan, 2009.

11. Sillanpaa, M. Micronutrient Assessment at Country Level: An International Study; Soils Bulletin No. 63; FAO: Rome, Italy, 1990; p. 208.

12. Hussain, A.; Zahir, Z.A.; Asghar, H.N.; Ahmad, M.; Jamil, M.; Naveed, M.; Akhtar, M.F.U.Z. Zinc solubilizing bacteria for zinc biofortification in cereals: A step toward sustainable nutritional security. In Role of Rhizospheric Microbes in Soil; Springer: Singapore, 2018; pp. 203-227.

13. Lopez-Millan, A.F.; Ellis, D.R.; Grusak, M.A. Effect of zinc and manganese supply on the activities of superoxide dismutase and carbonic anhydrase in Medicago truncatula wild type and raz mutant plants. Plant Sci. 2005, 168, 1015-1022. [CrossRef]

14. Sinclair, S.A.; Krämer, U. The zinc homeostasis network of land plants. Biochim. Biophys. Acta (BBA) Mol. Cell Res. 2012, 1823, 1553-1567. [CrossRef]

15. Bhatt, R.; Kukal, S.S.; Busari, M.A.; Arora, S.; Yadav, M. Sustainability issues on rice-wheat cropping system. Int. Soil Water Conserv. Res. 2016, 4, 64-74. [CrossRef]

16. Jat, H.S.; Datta, A.; Sharma, P.C.; Kumar, V.; Yadav, A.K.; Choudhary, M.; Choudhary, V.; Gathala, M.K.; Sharma, D.K.; Jat, M.L.; et al. Assessing soil properties and nutrient availability under conservation agriculture practices in a reclaimed sodic soil in cereal-based systems of North-West India. Arch. Agron. Soil Sci. 2018, 64, 531-545. [CrossRef]

17. Nawaz, A.; Farooq, M.; Lal, R.; Rehman, A.; Rehman, H. Comparison of conventional and conservation rice-wheat systems in Punjab. Pakistan. Soil Tillage Res. 2017, 169, 35-43. [CrossRef]

18. Nawaz, A.; Farooq, M.; Lal, R.; Rehman, A.; Hussain, T.; Nadeem, A. Influence of sesbania brown manuring and rice residue mulch on soil health, weeds and system productivity of conservation rice-wheat systems. Land Degrad. Develop. 2017, 28, 1078-1090. [CrossRef]

19. Zulfiqar, U.; Maqsood, M.; Hussain, S.; Anwar-ul-Haq, M. Iron nutrition improves productivity, profitability and biofortification of bread wheat under conventional and conservation tillage systems. J. Soil Sci. Plant Nutr. 2020. [CrossRef] 
20. Kumar, D. Effect of Conservation Agriculture on Vertical Distribution of Organic Carbon and Zinc Transformations under Alluvial Soil. Ph.D. Thesis, Department of Soil Science and Agricultural Chemistry, BAU, Sabour, India, 2017.

21. Loke, P.F.; Kotzé, E.; Du Preez, C.C. Impact of long-term wheat production management practices on soil acidity, phosphorus and some micronutrients in a semi-arid Plinthosol. Soil Res. 2013, 51, 415-426. [CrossRef]

22. Nawaz, A.; Farooq, M.; Nadeem, F.; Siddique, K.H.M.; Lal, R. Rice-wheat cropping systems in South Asia: Issues, options and opportunities. Crop Pasture Sci. 2019, 70, 395-427. [CrossRef]

23. Dixit, A.K.; Agrawal, R.K.; Das, S.K.; Sahay, C.S.; Choudhary, M.; Rai, A.K.; Palsaniya, D.R. Soil properties, crop productivity and energetics under different tillage practices in fodder sorghum + cowpea-wheat cropping system. Arch. Agron. Soil Sci. 2019, 65, 492-506. [CrossRef]

24. Naresh, R.K.; Timsina, J.; Bhaskar, S.; Gupta, R.K.; Singh, A.K.; Dhaliwal, S.S.; Rathore, R.S.; Kumar, V.; Singh, P.; Singh, S.P.; et al. Effects of tillage, residue and nutrient management on soil organic carbon dynamics and its fractions, soil aggregate stability and soil carbon sequestration: A review. EC Nutr. 2017, 12, 53-80.

25. Dasappagol, A.; Bellakki, M.A.; Ravi, M.V.; Kuchanur, P.H.; Jat, M.L. Distribution of zinc fractions in surface alfisol after five years of conservation agriculture practices in rainfed Pigeonpea. Int. J. Chem. Stud. 2017, 5, 227-232.

26. Dubock, A. An overview of agriculture, nutrition and fortification, supplementation and biofortification: Golden Rice as an example for enhancing micronutrient intake. Agric. Food Secur. 2017, 6, 1-20. [CrossRef]

27. Zulfiqar, U.; Maqsood, M.; Hussain, S. Biofortification of Rice with Iron and Zinc: Progress and Prospects. In Rice Research for Quality Improvement: Genomics and Genetic Engineering; Springer: Singapore, 2020; pp. 605-627.

28. Jaffe, G. Regulating transgenic crops: A comparative analysis of different regulatory processes. Transgenic Res. 2004, 13, 5-19. [CrossRef] [PubMed]

29. Saha, S.; Mandal, B.; Hazra, G.C.; Dey, A.; Chakraborty, M.; Adhikari, B.; Mukhopadhyay, S.K.; Sadhukhan, R. Can agronomic biofortification of zinc be benign for iron in cereals? J. Cereal Sci. 2015, 65, 186-191. [CrossRef]

30. Zulfiqar, U.; Hussain, S.; Ishfaq, M.; Ali, N.; Yasin, M.U.; Ali, M.A. Foliar manganese supply enhances crop productivity, net benefits, and grain manganese accumulation in direct-seeded and puddled transplanted rice. J. Plant Growth Regul. 2020, 1-18. [CrossRef]

31. Chattha, M.U.; Hassan, M.U.; Khan, I.; Chattha, M.B.; Mahmood, A.; Nawaz, M.; Subhani, M.N.; Kharal, M.; Khan, S. Biofortification of wheat cultivars to combat zinc deficiency. Front. Plant Sci. 2017, 8, 281. [CrossRef]

32. Dhaliwal, S.S.; Ram, H.; Shukla, A.K.; Mavi, G.S. Zinc biofortification of bread wheat, triticale, and durum wheat cultivars by foliar zinc fertilization. J. Plant Nutr. 2019, 42, 813-822. [CrossRef]

33. Hassan, M.U.; Chattha, M.U.; Ullah, A.; Khan, I.; Qadeer, A.; Aamer, M.; Khan, A.U.; Nadeem, F.; Khan, T.A. Agronomic biofortification to improve productivity and grain $\mathrm{Zn}$ concentration of bread wheat. Int. J. Agric. Biol. 2019, 21, 615-620.

34. FAO (Food and Agriculture Organization). World reference base for soil resources 2014. In International Soil Classification System for Naming Soils and Creating Legends for Soil Maps; FAO (Food and Agriculture Organization): Rome, Italy, 2014.

35. USDA (United State Department of Agriculture). Keys to soil taxonomy. In Natural Resources Conservation Service, 12th ed.; USDA (United State Department of Agriculture): Kansas City, MO, USA, 2014.

36. ISTA. International Rules for Seed Testing; International Seed Testing Association: Zürich, Switzerland, 2015.

37. Meier, U. Growth Stages of Mono-And Dicotyledonous Plants; Federal Biological Research Centre for Agriculture and Forestry: Berlin, Germany, 2001.

38. Blake, G.H.; Hartge, K.H. Bulk density. In Methods of Soil Analysis, 2nd ed.; Klute, A., Ed.; Agron. No. 9, Part; American Society of Agronomy: Madison, WI, USA, 1986; pp. 363-375.

39. Vomocil, J.A. Porosity. In Methods of Soil Analysis; Blake, C.A., Ed.; American Society of Agronomy: Madison, WI, USA, 1965; pp. 299-314.

40. Bremner, J.M.; Mulvaney, C.S. Total nitrogen. In Methods of Soil Analysis; Page, A.L., Miller, R.H., Keeny, D.R., Eds.; American Society of Agronomy: Madison, WI, USA, 1982; pp. 1119-1123.

41. Olsen, S.R. Estimation of Available Phosphorus in Soils by Extraction with Sodium Bicarbonate (No. 939); US Department of Agriculture: Madison, WI, USA, 1954.

42. Richards, L.A. Diagnosis and Improvement of Saline and Alkali Soils; LWW: New York, NY, USA, 1954; p. 154. 
43. Walkley, A.; Black, I.A. An examination of the Degtjareff method for determining soil organic matter, and a proposed modification of the chromic acid titration method. Soil Sci. 1934, 37, 29-38. [CrossRef]

44. Brookes, P.C.; Landman, A.; Pruden, G.; Jenkinson, D.S. Chloroform fumigation and the release of soil nitrogen: A rapid direct extraction method to measure microbial biomass nitrogen in soil. Soil Biol. Biochem. 1985, 17, 837-842. [CrossRef]

45. Anderson, J.M.; Ingram, J.S.I. Tropical Soil Biology and Fertility. In A Handbook of Methods, 2nd ed.; CAB International: Wallingford, UK, 1993.

46. Rashid, A. Mapping Zinc Fertility of Soils Using Indicator Plants and Soil Analyses. Ph.D. Thesis, University of Hawaii at Manoa, Monoa, HI, USA, 1986.

47. Haug, W.; Lantzsch, H. Sensitive method for the rapid determination of phytate in cereals and cereal products. J. Sci. Food Agric. 1983, 34, 1423-1424. [CrossRef]

48. Rehman, A.; Farooq, M.; Naveed, M.; Nawaz, A.; Shahzad, B. Seed priming of Zn with endophytic bacteria improves the productivity and grain biofortification of bread wheat. Eur. J. Agron. 2018, 94, 98-107. [CrossRef]

49. Rehman, A.; Farooq, M.; Naveed, M.; Ozturk, L.; Nawaz, A. Pseudomonas-aided zinc application improves the productivity and biofortification of bread wheat. Crop Pasture Sci. 2018, 69, 659-672. [CrossRef]

50. Miller, L.V.; Krebs, N.F.; Hambidge, K.M. A mathematical model of zinc absorption in humans as a function of dietary zinc and phytate. J. Nutr. 2007, 137, 135-141. [CrossRef] [PubMed]

51. Hambidge, K.M.; Miller, L.V.; Westcott, J.E.; Sheng, X.; Krebs, N.F. Zinc bioavailability and homeostasis. Am. J. Clin. Nutr. 2010, 91, 1478-1483. [CrossRef] [PubMed]

52. FAO (Food and Agriculture Organization). Food supply database 2007. In Food and Agriculture Organization; FAO (Food and Agriculture Organization): Rome, Italy, 2014; Available online: http:/faostat.fao.org/site/609/ default.aspx\# (accessed on 18 April 2018).

53. Fageria, N.K. The Use of Nutrients in Crop Plants; CRC Press: Boca Raton, FL, USA, 2009.

54. Shivay, Y.S.; Prasad, R. Zinc-coated urea improves productivity and quality of basmati rice (Oryza sativa L.) under zinc stress condition. J. Plant Nutr. 2012, 35, 928-951. [CrossRef]

55. CIMMYT Economics Program, International Maize, \& Wheat Improvement Center. From Agronomic Data to Farmer Recommendations: An Economics Training Manual (No. 27); CIMMYT: Texcoco, Mexico, 1988; pp. 31-33.

56. Steel, R.G.D.; Torrie, J.H.; Dickey, D.A. Principles and Procedures of Statistics a Biometrical Approach (No. 519.5 S8); McGraw-Hill: New York, NY, USA, 1997.

57. Shah, A.N.; Tanveer, M.; Shahzad, B.; Yang, G.; Fahad, S.; Ali, S.; Bukhari, M.A.; Tung, S.A.; Hafeez, A.; Souliyanonh, B. Soil compaction effects on soil health and crop productivity: An overview. Environ. Sci. Pollut. Res. 2017, 24, 10056-10067. [CrossRef] [PubMed]

58. Hobbs, P.R. Conservation agriculture: What is it and why is it important for future sustainable food production? J. Agric. Sci. 2007, 145, 127-137. [CrossRef]

59. Meena, J.R.; Behera, U.K.; Chakraborty, D.; Sharma, A.R. Tillage and residue management effect on soil properties, crop performance and energy relations in green gram (Vigna radiata L.) under maize-based cropping systems. Int. Soil Water Cons. Res. 2015, 3, 261-272. [CrossRef]

60. Busari, M.A.; Kukal, S.; Kaur, A.; Bhatt, R.; Dulazi, A.A. Conservation tillage impacts on soil, crop and the environment. Int. Soil Water Cons. Res. 2015, 3, 119-129. [CrossRef]

61. Nandan, R.; Singh, V.; Singh, S.S.; Kumar, V.; Hazra, K.K.; Nath, C.P.; Pooniad, S.; Malikd, R.K.; Bhattacharyyae, R.; McDonald, A. Impact of conservation tillage in rice-based cropping systems on soil aggregation, carbon pools and nutrients. Geoderma 2019, 340, 104-114. [CrossRef]

62. Chen, H.; Hou, R.; Gong, Y.; Li, H.; Fan, M.; Kuzyakov, Y. Effects of 11 years of conservation tillage on soil organic matter fractions in wheat monoculture in Loess Plateau of China. Soil Tillage Res. 2009, 106, 85-94. [CrossRef]

63. Lupwayi, N.; Hanson, K.; Harker, K.; Clayton, G.; Blackshaw, R.; O’Donovan, J.; Johnson, E.; Gan, Y.; Irvine, R.; Monreal, M. Soil microbial biomass, functional diversity and enzyme activity in glyphosate-resistant wheat-canola rotations under low-disturbance direct seeding and conventional tillage. Soil Biol. Biochem. 2007, 39, 1418-1427. [CrossRef]

64. Balota, E.L.; Colozzi-Filho, A.; Andrade, D.S.; Dick, R.P. Microbial biomass in soils under different tillage and crop rotation systems. Biol. Fert. Soils. 2003, 38, 15-20. [CrossRef] 
65. Haddaway, N.R.; Hedlund, K.; Jackson, L.E.; Kätterer, T.; Lugato, E.; Thomsen, I.K.; Jørgensen, H.B.; Isberg, P.E. How does tillage intensity affect soil organic carbon? A systematic review. Environ. Evid. 2016, 6, 30. [CrossRef]

66. Zikeli, S.; Gruber, S.; Teufel, C.F.; Hartung, K.; Claupein, W. Effects of reduced tillage on crop yield, plant available nutrients and soil organic matter in a 12-year long-term trial under organic management. Sustainability 2013, 5, 3876-3894. [CrossRef]

67. Roscoe, R.; Burman, P. Tillage effects on soil organic matter in the density fractions of a Cerrado Oxisol. Soil Tillage Res. 2003, 70, 107-119. [CrossRef]

68. Paul, B.K.; Vanlauwe, B.; Ayuke, F.; Gassner, A.; Hoogmoed, M.; Hurisso, T.T.; Koala, S.; Lelei, D.; Ndabamenye, T.; Six, J.; et al. Medium-term impact of tillage and residue management on soil aggregate stability, soil carbon, and crop productivity. Agric. Ecosyst. Environ. 2013, 164, 14-22. [CrossRef]

69. Das, A.; Lyngdoh, D.; Ghosh, P.K.; Lal, R.; Layek, J.; Idapuganti, R.G. Tillage and cropping sequence effect on physico-chemical and biological properties of soil in Eastern Himalayas, India. Soil Tillage Res. 2018, 180, 182-193. [CrossRef]

70. Meisinger, J.J.; Palmer, R.E.; Timlin, D.J. Effects of tillage practices on drainage and nitrate leaching from winter wheat in the Northern Atlantic Coastal-Plain USA. Soil Tillage Res. 2015, 151, 18-27. [CrossRef]

71. Ali, M.A.; Ladha, J.K.; Rickman, J.; Lales, J.S. Comparison of different methods of rice establishment and nitrogen management strategies for lowland rice. J. Crop Improv. 2006, 16, 173-189. [CrossRef]

72. Vincent, A.G.; Turner, B.L.; Tanner, E.V.J. Soil organic phosphorus dynamics following perturbation of litter cycling in a tropical moist forest. Eur. J. Soil Sci. 2010, 61, 48-57. [CrossRef]

73. Martin-Rueda, I.; Munoz-Guerra, L.M.; Yunta, F.; Esteban, E.; Tenorio, J.L.; Lucena, J.J. Tillage and crop rotation effects on barley yield and soil nutrients on a Calciortidic Haploxeralf. Soil Tillage Res. 2007, 92, 1-9. [CrossRef]

74. Bam, R.K.; Kumaga, F.K.; Ori, K.; Asiedu, E.A. Germination, vigour and dehydrogenase activity of naturally aged rice (Oryza sativa L.) seeds soaked in potassium and phosphorus. Asian J. Plant Sci. 2006, 5, 948-955.

75. Singh, H.; Jassal, R.K.; Kang, J.S.; Sandhu, S.S.; Kang, H.; Grewal, K. Seed priming techniques in field crops-A review. Agric. Rev. 2015, 36, 251-264. [CrossRef]

76. Farooq, M.; Usman, M.; Nadeem, F.; Rehman, H.; Wahid, A.; Basra, S.M.A.; Siddique, K.H.M. Seed priming in field crops: Potential benefits, adoption and challenges. Crop Pasture Sci. 2019, 70, 731. [CrossRef]

77. Rehman, A.; Farooq, M.; Ozturk, L.; Asif, M.; Siddique, K.H.M. Zinc nutrition in wheat-based cropping systems. Plant Soil 2018, 422, 283-315. [CrossRef]

78. Reis, S.; Pavia, I.; Carvalho, A.; Moutinho-Pereira, J.; Correia, C.; Lima-Brito, J. Seed priming with iron and zinc in bread wheat: Effects in germination, mitosis and grain yield. Protoplasma 2018, 255, 1179-1194. [CrossRef]

79. Bityutskii, N.P.; Davydovskaya, E.N.; Malyuga, E.A.; Yakkonen, K.L. Mechanisms underlying iron and zinc transport to axis organs in grain during early seedling development of maize. J. Plant Nutr. 2004, 27, 1525-1541. [CrossRef]

80. Cakmak, I.; Marschner, H. Effect of zinc nutritional status on activities of superoxide radical and hydrogen peroxide scavenging enzymes in bean leaves. Plant Soil 1993, 155, 127-130. [CrossRef]

81. Mabesa, R.L.; Impa, S.M.; Grewal, D.; Johnson-Beebout, S.E. Contrasting grain-Zn response of biofortification rice (Oryza sativa L.) breeding lines to foliar Zn application. Field Crop. Res. 2013, 149, 223-233. [CrossRef]

82. Wu, C.Y.; Lu, L.L.; Yang, X.E.; Feng, Y.; Wei, Y.Y.; Hao, H.L.; Stoffella, P.J.; He, Z.L. Uptake, translocation, and remobilization of zinc absorbed at different growth stages by rice genotypes of different $\mathrm{Zn}$ densities. J. Agric. Food Chem. 2010, 58, 6767-6773. [CrossRef] [PubMed]

83. Li, M.; Yang, X.W.; Tian, X.H.; Wang, S.X.; Chen, Y.L. Effect of nitrogen fertilizer and foliar zinc application at different growth stages on zinc translocation and utilization efficiency in winter wheat. Cereal Res. Commun. 2014, 42, 81-90. [CrossRef]

84. Farooq, M.; Ullah, A.; Rehman, A.; Nawaz, A.; Nadeem, A.; Wakeel, A.; Nadeem, F.; Siddique, K.H.M. Application of zinc improves the productivity and biofortification of fine grain aromatic rice grown in dry seeded and puddled transplanted production systems. Field Crop. Res. 2018, 216, 53-62. [CrossRef]

85. Dimkpa, C.O.; White, J.C.; Elmer, W.H.; Gardea-Torresdey, J. Nanoparticle and ionic Zn promote nutrient loading of sorghum grain under low NPK fertilization. J. Agric. Food Chem. 2017, 65, 8552-8559. [CrossRef] [PubMed] 
86. Gupta, R.K.; Gangoliya, S.S.; Singh, N.K. Reduction of phytic acid and enhancement of bioavailable micronutrients in food grains. J. Food Sci. Technol. 2015, 52, 676-684. [CrossRef]

87. Huang, C.; Barker, S.J.; Langridge, P.; Smith, F.W.; Graham, R.D. Zinc deficiency up-regulates expression of high-affinity phosphate transporter genes in both phosphate- sufficient and-Deficient barley roots. Plant Physiol. 2000, 124, 415-422. [CrossRef]

88. Cakmak, I.; Pfeiffer, W.H.; McClafferty, B. Biofortification of durum wheat with zinc and iron. Cereal Chem. 2010, 87, 10-20. [CrossRef]

89. Cakmak, I.; Kalayci, M.; Kaya, Y.; Torun, A.A.; Aydin, N.; Wang, Y.; Arisoy, Z.; Erdem, H.; Yazici, A.; Gokmen, O.; et al. Biofortification and localization of zinc in wheat grain. J. Agric. Food Chem. 2010, 58, 9092-9102. [CrossRef]

Publisher's Note: MDPI stays neutral with regard to jurisdictional claims in published maps and institutional affiliations.

(C) 2020 by the authors. Licensee MDPI, Basel, Switzerland. This article is an open access article distributed under the terms and conditions of the Creative Commons Attribution (CC BY) license (http://creativecommons.org/licenses/by/4.0/). 